\title{
On the Lipschitz regularity and asymptotic behaviour of the free boundary for classes of minima of inhomogeneous two-phase Alt-Caffarelli functionals in Orlicz spaces
}

\author{
J. Ederson M. Braga ${ }^{1}$
}

Received: 1 May 2017 / Accepted: 28 April 2018 / Published online: 9 May 2018

(C) Fondazione Annali di Matematica Pura ed Applicata and Springer-Verlag GmbH Germany, part of Springer Nature 2018

Abstract In this paper, we study classes of minimizers of inhomogeneous two-phase AltCaffarelli functionals of the type

$$
\mathcal{J}_{G}(u, \Omega)=\int_{\Omega}\left[G(|\nabla u|)+f_{1}(x) H_{1}\left(u^{+}\right)+f_{2}(x) H_{2}\left(u^{-}\right)+Q\left(h_{1}, h_{2}\right)(u)(x)\right] \mathrm{d} x,
$$

on a bounded domain $\Omega \subset \mathbb{R}^{n}$, where $G, H_{1}$ and $H_{2}$ are power-like $N$-functions, $f_{1}, f_{2} \in$ $L^{q}(\Omega)$ for suitable $n \leq q \leq \infty$, and $h_{1}, h_{2} \in L^{\infty}(\Omega)$. Hölder and non-degeneracy estimates for minima are obtained and in the particular case where such minimizers are weak solutions of non-singular PDEs we provide log-Lipschitz type estimates. In the sequel, since the AltCaffarelli-Friedman monotonicity formula is missing in our context and there is a mistake in a proof of Lipschitz continuity for minimizers in Zheng et al. (Monatsh Math 172:441475, 2013), we extend the results of Braga et al. (Ann Inst H Poincaré Anal Non Linéaire 31(4):823-850, 2014) establishing the Lipschitz regularity for more general class of minima under the additional condition of small Lebesgue density on one of the phases along the free boundary. We finish this paper with a result that establishes density estimates from below for the positive and negative phase on points inside the contact set between the free boundaries in the case where minimizers are not Lipschitz. Such estimates allow us to provide a preliminary full description of the free boundary for any minima even if the Lipschitz regularity (as optimal regularity) is unknown.

Keywords Free boundary problems · Lipschitz regularity · Minimizers · Orlicz spaces · Degenerate/singular equations

Mathematics Subject Classification $35 \mathrm{~J} 60 \cdot 35 \mathrm{~J} 70 \cdot 35 \mathrm{~J} 75 \cdot 35 \mathrm{R} 35$

J. Ederson M. Braga edersonbraga@mat.ufc.br

1 Departamento de Matemática, Universidade Federal do Ceará, Campus do Pici - Bloco 914, Fortaleza, Ceará CEP 60455-760, Brazil 


\section{Introduction}

In the beginning of the $80 \mathrm{~s} \mathrm{W.} \mathrm{Alt} \mathrm{and} \mathrm{L.} \mathrm{Caffarelli} \mathrm{investigated} \mathrm{in} \mathrm{[2]} \mathrm{the} \mathrm{following} \mathrm{problem}$

$$
\min _{u \in K_{\varphi}}\left\{\int_{\Omega}\left[|\nabla u|^{2}+Q(x) \chi_{\{u>0\}}\right] \mathrm{d} x\right\}, \quad K_{\varphi}:=\left\{u \in H^{1}(\Omega): u-\varphi \in H_{0}^{1}(\Omega)\right\} .
$$

They proved that solutions of the above minimization problem are nonnegative Lipschitz functions and weak solutions of the one-phase Free Boundary Problem (FBP):

$$
\begin{cases}\Delta u=0 & \text { in }\{u>0\} \cap \Omega, \\ \left|\nabla u^{+}\right|=Q & \text { on } F^{+}(u):=\partial\{u>0\} \cap \Omega, \\ u=\varphi & \text { on } \partial \Omega,\end{cases}
$$

for $0 \leq \varphi \in H^{1}(\Omega) \cap L^{\infty}(\partial \Omega)$ and $0<\lambda \leq Q(x) \leq \Lambda$. Particularly, (1.2) ensures that Lipschitz is the best regularity for minima. Still from (1.2) another interesting issue stands out in these work. More precisely, what is the regularity of the (a priori unknown) interface $F^{+}(u)$ ? In this way the authors show that if $Q$ is sufficiently regular, then $F^{+}(u)$ (so-called free boundary of $u$ ) is a $C^{1, \alpha}$-regular surface near "flat" free boundary points.

Few years later, Alt, Caffarelli together with A. Friedman extended the problem above in the papers [3,4]. In [3] they study a similar functional as in (1.1) related to suitable quasilinear equations (still in the one-phase scenario), and in [4] consider a minimization problem where minimizers may change of sign (originating so two-phase FBPs). Although in $[3,4]$ the authors have followed a similar way as in [2] for the two-phase problem, a new ingredient needed to be developed: the well-known Alt-Caffarelli-Friedman monotonicity formula. With such powerful tool, they showed that the gradient of minima are bounded in the neighbourhood of the free boundary and hence the local Lipschitz regularity of minimizers and the regularity of the free boundary have been achieved.

Only from 2005 the (one-phase) Alt-Caffarelli minimum problem was extended for singular/degenerate type operators (see, for instance $[9,18]$ ). In these papers, the energy is substituted, respectively, by p-energy, $\int_{\Omega}|\nabla u|^{p} \mathrm{~d} x, 1<p<\infty$, and $G$-energy, $\int_{\Omega} G(|\nabla u|) \mathrm{d} x$, where $G$ is a suitable $N$-function. ${ }^{1}$ By a new approach using indirect arguments and compactness results, similar theorems about the optimal regularity of minima and properties of the free boundary were recovered. As in [2], the minimizers in $[9,18]$ are also weak solutions of a FBP as (1.2).

For two-phase setting, the situation is much more delicate and less understood in the general context. To the best of our knowledge, up to the present time and except in those cases where a variant of the ACF monotonicity formula exists, for example [4,7], two-phase minimization problems have only partial results. In fact, for singular/degenerate operators see $[6,11]$ (homogeneous case), and [15] (inhomogeneous case for non-singular p-Laplace operator, i.e., $p \geq 2$ ). However, in 2013, in order to answer in a complete form the two main issues in a two-phase scenario (in the absence of the ACF monotonicity formula) for non-singular type operators ${ }^{2}$ in the Orlicz context, the authors in [20] studied minimizers of

$$
\mathcal{J}(u)=\int_{\Omega}\left[G(|\nabla u|)+\lambda_{+} \chi_{\{u>0\}}+\lambda_{-} \chi_{\{u<0\}}+f u\right] \mathrm{d} x,
$$

where $G^{\prime}(t) / t$ is nondecreasing, $f \in L^{\infty}(\Omega)$ and $0 \leq \lambda_{-}<\lambda_{+}<\infty$. In these work, the statement of Theorem 1.3 ensures that minimizers of (1.3) are locally Lipschitz continuous.

\footnotetext{
1 A definition for $N$-functions is presented in the next page.

2 For non-singular operators see Sect. 8.
} 
But, unfortunately, there exists a mistake in a proof of this result. More precisely, the interior $C^{1, \alpha}$ regularity of the solutions to $\operatorname{div}\left(\frac{g(|\nabla u|)}{|\nabla u|} \nabla u\right)=0$, where $g=G^{\prime}$, in bounded domains $\Omega \subset \mathbb{R}^{n}$, was used incorrectly. In fact, the authors consider a ball $B_{r} \Subset \Omega$ and the following Dirichlet problem

$$
\begin{cases}\operatorname{div}\left(\frac{g(|\nabla h|)}{|\nabla h|} \nabla h\right)=0 & \text { in } B_{r}, \\ h=u_{0} & \text { on } \partial B_{r},\end{cases}
$$

where $u_{0}$ is a minimizer to (1.3). Since, a priori, $u_{0}$ is only log-Lipschitz continuous and $h$ is not defined in $\Omega \backslash \bar{B}_{r}$ it is impossible to conclude that $\nabla h$ is bounded in $B_{r}$. We would need a Lipschitz estimate up to the boundary for $h$ with a prescribed log-Lipschitz boundary data. However, we observe that if $G(t)=\frac{1}{2} t^{2}$ and $e_{1}=(1,0, \cdots, 0)$,

$$
\begin{cases}\Delta h=0 & \text { in } B_{1}\left(e_{1}\right) \\ h(x)=|x| \cdot|\log | x|| & \text { on } \partial B_{1}\left(e_{1}\right)\end{cases}
$$

gives us a simple counterexample. In fact, $\lim _{t \rightarrow 0^{+}}\left|\nabla h\left(t e_{1}\right)\right|=+\infty$.

In view of all the considerations above, the objective of the present manuscript is to extend one more time this theory in the two-phase setting to the context of Orlicz-Sobolev spaces. More precisely, for $\Omega \subset \mathbb{R}^{n}, n \geq 2$, a bounded domain with Lipschitz boundary, $G, H_{1}, H_{2}:[0, \infty) \longrightarrow[0, \infty)$ suitable $N$-functions, $f_{1}, f_{2} \in L^{q}(\Omega), n \leq q \leq \infty$ and $h_{1}, h_{2} \in L^{\infty}(\Omega)$, we investigate the optimal regularity and geometric properties for local and global minimizers of classes of functionals of the type

$$
\mathcal{J}_{G}(u, \Omega)=\int_{\Omega}\left[G(|\nabla u|)+f_{1}(x) H_{1}\left(u^{+}\right)+f_{2}(x) H_{2}\left(u^{-}\right)+Q\left(h_{1}, h_{2}\right)(u)(x)\right] \mathrm{d} x,
$$

where

$$
Q\left(h_{1}, h_{2}\right)(u)(x):=h_{2}(x) \cdot \chi_{\{u>0\}}+h_{1}(x) \cdot \chi_{\{u<0\}}+\min \left(h_{1}(x), h_{2}(x)\right) \cdot \chi_{\{u=0\}} .
$$

In addition, we observe that fixed a boundary datum $\varphi \in W^{1, G}(\Omega) \cap L^{\infty}(\partial \Omega)$, if $G$, $H_{i}$ and $h_{i}$ are sufficiently regular, at least formally, the Euler-Lagrange equations for such minimizers are general FBPs of the type

$$
\begin{cases}\mathcal{L}_{g} u=f_{1} H_{1}^{\prime}\left(u^{+}\right)+f_{2} H_{2}^{\prime}\left(u^{-}\right) & \text {in }\left(\{u>0\} \cup\{u \leq 0\}^{0}\right) \cap \Omega, \\ \Gamma_{G}\left(\left|\nabla u^{+}\right|\right)-\Gamma_{G}\left(\left|\nabla u^{-}\right|\right)=h_{2}-h_{1} & \text { on } F^{ \pm}(u):=(\partial\{u>0\} \cup \partial\{u<0\}) \cap \Omega, \\ u=\varphi & \text { on } \partial \Omega,\end{cases}
$$

where

$$
\mathcal{L}_{g} u:=\operatorname{div}\left(\frac{g(|\nabla u|)}{|\nabla u|} \cdot \nabla u\right), \quad g(t)=G^{\prime}(t) \quad \text { and } \quad \Gamma_{G}(t)=g(t) t-G(t) .
$$

Here, we remember that $G$ is a $N$-function if $G(t)=\int_{0}^{t} g(s) \mathrm{d} s$, where $g:[0, \infty) \rightarrow \mathbb{R}$ is a positive nondecreasing function such that $g(0)=0, \lim _{t \rightarrow \infty} g(t)=\infty$ and $g$ is right continuous, that is, if $t \geq 0$ then $\lim _{s \rightarrow t+} g(s)=g(t)$. Basics properties and results in Orlicz Spaces theory can be found in [1].

This paper is a extension of the results obtained in [6], as we now explain. Here, we consider functionals such that the Euler-Lagrange equations related are inhomogeneous. We obtain non-degeneracy for minimizers in the neighbourhood of the free boundary and logLipschitz type estimates for minima of non-singular operators (see definition in Sect. 8). We observe that such results were not presented in [6]. We also extend the compactness result 
about $N$-functions (Theorem 6.1 of [6]). For this, we weaken the Morrey $\beta$-control, namely, if $g=G^{\prime}$ and $Q_{g}(t)=\frac{t g^{\prime}(t)}{g(t)}$,

$$
\int_{t}^{t+\kappa}\left|Q_{g}^{\prime}(t)\right| \mathrm{d} t \leq C\left(\delta, g_{0}, \beta\right)\left(\frac{\kappa}{t}\right)^{\beta}
$$

for more general modulus of continuity for $Q_{g}$. For instance, a Dini type control,

$$
\int_{0}^{L-l} \frac{\omega_{g}^{l, L}(t)}{t} \mathrm{~d} t \leq C\left(\delta, g_{0}\right) \cdot \xi_{1}\left(\frac{L}{l}\right) \cdot \xi_{2}\left(\frac{L-l}{l}\right),
$$

for a modulus of continuity $\omega_{g}^{l, L}(t)$ of $Q_{g}$ and some functions $\xi_{i}:(0, \infty) \rightarrow(0, \infty)$ (for more details see Definition 2.1). We point out that the Morrey $\beta$-control is a key ingredient in [6] to prove the Lipschitz continuity of minimizers under universal smallness of the density of the negative phase. We emphasize that the main contribution in this paper is to prove the main results of [6] for such Dini type modulus of continuity for $Q_{g}$, precisely, the Lipschitz regularity of minima under small density on one of the phases and universal asymptotic density estimates from below for the sets $\{u<0\}$ and $\{u>0\}$ for some possible minimizer $u \notin C_{l o c}^{0,1}$. Finally, even in the homogeneous case, we observe that our main results are slightly more general than those obtained in [6] (see Theorems 2.2, 2.3 and 2.4 in the next section).

Let us now discuss the precise conditions that will be assumed throughout the remainder of the paper. For singular/degenerate elliptic operators, we will assume the same conditions as in [17]. In this work, G. Lieberman studies the regularity theory for equations of the type $\mathcal{L}_{g} u=B(x, u, \nabla u)$. The conditions we refer to are:

\section{- Primitive Condition:}

$$
G^{\prime}(t)=g(t), \text { where } g \in C^{0}([0,+\infty)) \cap C^{1}((0,+\infty)) ;
$$

- Quotient Condition: for $0<\delta \leq g_{0}$ fixed constants,

$$
0<\delta \leq Q_{g}(t):=\frac{\operatorname{tg}^{\prime}(t)}{g(t)} \leq g_{0}, \quad \forall t>0 .
$$

In accordance with [18], Lieberman's condition (PC) and (QC) assure that $\mathcal{L}_{g} u=$ $B(x, u, \nabla u)$ is equivalent to a uniformly elliptic equation (in non-divergence form) with (positive) ellipticity constants $\lambda=\min \{1, \delta\}$ and $\Lambda=\max \left\{1, g_{0}\right\}$ in the sets $\{|\nabla u| \geq c\}$, for any $c>0$ (Remark 7.1, [18]). Such conditions do not imply any kind of homogeneity for the function $G$. Moreover, it allows different behaviours for $g$ when $|\nabla u|$ is close to zero or infinity. This lack of homogeneity will result in further difficulties in obtaining certain estimates. It is also worth emphasizing the large number of examples of functions satisfying (PC) and (QC), namely, $g(t)=t^{p}$ with $\delta=g_{0}=p, p>0 ; g(t)=a t^{p}+b t^{p^{\prime}}$ where $a, b, p, p^{\prime}>0$ with $\delta=\min \left\{p, p^{\prime}\right\}$ and $g_{0}=\max \left\{p, p^{\prime}\right\}$ and $g(t)=t^{p} \log (a t+b)$ for $p, a, b>0$ where in this case $\delta=p$ and $g_{0}=p+1$. Many others examples are presented in $[6,18,20]$. We also observe that FBPs as (1.5) appear in many applications, in particular, in the study of the flow of two liquids in models of jets and cavities.

For the $N$-functions $H_{i}:[0, \infty) \longrightarrow[0, \infty)$, we assume that $H_{i} \in C^{1}[0, \infty)$ and there exist constants $0 \leq p_{i} \leq \rho_{i}<\infty$ and $\gamma_{i}>0$ such that

$$
H_{i}(t) \leq \gamma_{i} \cdot \max \left\{t^{1+p_{i}}, t^{1+\rho_{i}}\right\}, \forall t \geq 0, i=1,2 .
$$


As mentioned above, in this paper, we deal with the inhomogeneous case for two-phase Alt-Caffarelli minimum type problem. Similarly as in [6], instead of focusing on any particular case, we would like to obtain universal estimates for the largest class of functionals that share the same universal and specific parameters. Once established the existence and bounds for minimizers of (1.4), we focus on appropriate classes of local minimizers to obtain regularity results, non-degeneracy estimates, suitable conditions for Lipschitz regularity and some geometry of the free boundaries. Therefore, we define a preliminary class of $N$-functions

$$
\mathcal{G}\left(\delta, g_{0}\right):=\{G:[0, \infty) \rightarrow[0, \infty) ; \quad G \text { is } N \text {-function satisfying }(P C) \text { and }(Q C)\} .
$$

This paper is organized as follows: In Sect. 2, we establish classes of the $N$-functions and functionals that we will systematically use along the paper. In this section, we also present our main results. Section 3 is dedicated to preliminary results in Orlicz-Sobolev spaces developed in $[6,17,18]$. Section 4 deals with the existence and $L^{\infty}$ estimates for global minimizers. Section 5 is devoted to the uniform $C_{l o c}^{0, \alpha}$ estimates for the class of local minimizers of functionals related to non-degenerate $N$-functions $G$, i.e. functionals where $G$ satisfies the following condition: there exists $\varepsilon_{0}>0$ such that $G(1) \geq \varepsilon_{0}, \forall G \in \mathcal{G}\left(\delta, g_{0}\right)$. In Sect. 6, we provide a proof for the local Lipschitz regularity for one-phase minimizers. In Sect. 7, we show the non-degeneracy properties for minima. In Sect. 8, we prove the uniform $\log$-Lipschitz regularity for minimizers in classes where $\delta \geq 1$, i.e. the minima are weak solutions of non-singular Euler-Lagrange equations. In Sect. 9, we address compactness and scaling results. The proof of our main result (Theorem 2.2) is discussed in Sect. 10. In Sect. 11, we discuss two applications of the Theorem 2.2: The Lipschitz regularity for two-phase minimizers under small density of one of the phases along the free boundary and density estimates from below for the positive and negative phase on points inside the contact set between the free boundaries in the case where a minimizer is not Lipschitz. We finish the section establish a preliminary description of the free boundary for any minima even in the case where Lipschitz regularity fails to be the optimal one.

\section{Some definitions and main results}

We start this section remember the definition of modulus of continuity. A modulus of continuity is a nondecreasing continuous function $\omega:[0, \infty) \rightarrow[0, \infty)$ where $\omega(0)=0$ and $\omega(t)>0, \forall t>0$. Now, for $G \in \mathcal{G}\left(\delta, g_{0}\right), Q_{g}$ as in (QC) and for any $0<l<L<\infty$, we define

$$
\omega_{g}^{l, L}(t):=\sup \left\{\left|Q_{g}(x)-Q_{g}(y)\right|: l \leq x, y \leq L \text { and }|x-y| \leq t\right\} .
$$

Definition 2.1 (Modulus of continuity for $Q_{g}$ ) We say that a $N$-function $G \in \mathcal{G}\left(\delta, g_{0}\right)$ belongs to:

(i) $\mathcal{G}_{\left(\xi_{1}, \xi_{2}\right) \cdot D}\left(\delta, g_{0}\right)$ if, for the nondecreasing functions $\xi_{1}, \xi_{2}:(0, \infty) \rightarrow(0, \infty)$ with $\lim _{t \rightarrow 0^{+}} \xi_{2}(t)=0$ and any $0<l<L<\infty$, the following Dini type control holds

$$
\int_{0}^{L-l} \frac{\omega_{g}^{l, L}(t)}{t} \mathrm{~d} t \leq C_{1}^{*}\left(\delta, g_{0}\right) \cdot \xi_{1}\left(\frac{L}{l}\right) \cdot \xi_{2}\left(\frac{L-l}{l}\right) .
$$


(ii) $\mathcal{G}_{\beta \cdot H}\left(\delta, g_{0}\right)$, for $0<\beta \leq 1$, if $g \in W_{l o c}^{2,1}((0,+\infty))$ and, for any $t>0$ and $\kappa>0$ the following $\beta$ - Hölder type control holds

$$
\int_{t}^{t+\kappa}\left|Q_{g}^{\prime}(s)\right| \mathrm{d} s \leq C_{2}^{*}\left(\delta, g_{0}, \beta\right) \cdot\left(\frac{\kappa}{t}\right)^{\beta} .
$$

(iii) $\mathcal{G}_{\text {Lip }}\left(\delta, g_{0}\right)$ if $g \in W_{l o c}^{2,1}((0,+\infty))$ and holds the Lipschitz type control

$$
0 \leq \frac{t^{2}\left|g^{\prime \prime}(t)\right|}{g(t)} \leq C_{3}^{*}\left(\delta, g_{0}\right), \text { for a.e. } t>0 .
$$

In Sect. 9, we verify that for every $\beta \in(0,1]$

$$
\mathcal{G}_{\text {Lip }}\left(\delta, g_{0}\right) \varsubsetneqq \mathcal{G}_{\beta \cdot H}\left(\delta, g_{0}\right) \varsubsetneqq \bigcup_{\gamma \in(0,1]} \mathcal{G}_{\gamma \cdot H}\left(\delta, g_{0}\right) \varsubsetneqq \mathcal{G}_{\left(\xi_{1}, \xi_{2}\right) \cdot D}\left(\delta, g_{0}\right) \subseteq \mathcal{G}\left(\delta, g_{0}\right) .
$$

In this moment, we observe that there may exist sequences of $N$-functions in $\mathcal{G}\left(\delta, g_{0}\right)$ that converge to zero in any compact subset of $[0, \infty)$. Indeed, consider, for example, $G_{N}(t)=$ $\frac{1}{N} t^{3}$. On the other hand, the graph of (one-phase local) minimizers of the functionals $\mathcal{J}_{G}$, when $f_{1}=f_{2} \equiv 0$, hits the free boundary with an angle comparable to negative powers of $G(1)$. In fact, if $G(1)$ is small enough

$$
C_{4}^{*} \cdot\left(\frac{1}{G(1)}\right)^{\frac{1}{1+g_{0}}} \leq|\nabla u| \leq C_{5}^{*} \cdot\left(\frac{1}{G(1)}\right)^{\frac{1}{1+\delta}} \text { along } F^{+}(u)=\partial\{u>0\} \cap \Omega,
$$

for some universal and positive constants $C_{4}^{*}, C_{5}^{*}$ (see Section 6 in [6]). In particular, if $u_{k}$ is a minimizer for $\mathcal{J}_{G_{k}}$ with $G_{k}(1) \searrow 0$, then $\left|\nabla u_{k}\right| \nearrow+\infty$. Thus, universal estimates in classes of functionals can only hold for non-degenerate subclasses of $\mathcal{G}\left(\delta, g_{0}\right)$, i.e, subclasses where $G(1)$ is bounded from below away from zero, say $G(1) \geq \varepsilon_{0}>0$. In order to ensure that $N$-functions belong to non-degenerate classes, we consider the following definition (see [6]):

Definition 2.2 (Non-degenerate subclasses) For $\varepsilon_{0}>0$ we define

$$
\begin{aligned}
& \mathcal{G}\left(\delta, g_{0}, \varepsilon_{0}\right)=\left\{G \in \mathcal{G}\left(\delta, g_{0}\right): G(1) \geq \varepsilon_{0}\right\} \quad \text { and } \\
& \mathcal{G}_{\left(\xi_{1}, \xi_{2}\right) \cdot D}\left(\delta, g_{0}, \varepsilon_{0}\right)=\left\{G \in \mathcal{G}_{\left(\xi_{1}, \xi_{2}\right) \cdot D}\left(\delta, g_{0}\right): G(1) \geq \varepsilon_{0}\right\} .
\end{aligned}
$$

Similarly we define $\mathcal{G}_{\beta \cdot H}\left(\delta, g_{0}, \varepsilon_{0}\right)$ and $\mathcal{G}_{\text {Lip }}\left(\delta, g_{0}, \varepsilon_{0}\right)$.

Now we define the main classes of functions in this paper.

Definition 2.3 We say that a function $u$ belongs to class $\mathcal{S}(\Omega)$ if $u$ is a minimizer of a functional

$$
\mathcal{J}_{G}(u, \Omega)=\int_{\Omega}\left[G(|\nabla u|)+f_{1}(x) H_{1}\left(u^{+}\right)+f_{2}(x) H_{2}\left(u^{-}\right)+Q\left(h_{1}, h_{2}\right)(u)(x)\right] \mathrm{d} x,
$$

for some $G \in \mathcal{G}\left(\delta, g_{0}\right)$ and $H_{i} \in C^{1}([0, \infty))$ satisfying (HC) for nonnegative constants $\gamma_{i}, p_{i}, \rho_{i}$, where $p_{i} \leq \rho_{i}<\delta$ and $\lambda_{i}$ and $\mu_{i}$, positive constants such that

$$
\left\|f_{i}\right\|_{L^{q}(\Omega)} \leq \lambda_{i}, \quad 0 \leq h_{i} \leq \mu_{i} .
$$


In fact, $\mathcal{S}(\Omega):=\mathcal{S}\left(\Omega, \delta, g_{0}, p_{i}, \rho_{i}, \gamma_{i}, \lambda_{i}, \mu_{i}\right)$.

If $G \in \mathcal{G}\left(\delta, g_{0}, \varepsilon_{0}\right)$, we say that $u \in \mathcal{S}_{\varepsilon_{0}}(\Omega)=: \mathcal{S}\left(\Omega, \delta, g_{0}, p_{i}, \rho_{i}, \gamma_{i}, \lambda_{i}, \mu_{i}, \varepsilon_{0}\right)$.

For $M>0$ we define

$$
\mathcal{S}_{\varepsilon_{0}}(\Omega, M):=\left\{u \in \mathcal{S}_{\varepsilon_{0}}(\Omega):\|u\|_{L^{\infty}(\Omega)} \leq M\right\} .
$$

We say that $u \in S_{\varepsilon_{0}}\left(\Omega, M, \Lambda_{i}^{M}\right)$, for the positive constant $\Lambda_{i}^{M}$, if $u \in \mathcal{S}_{\varepsilon_{0}}(\Omega, M)$ and

$$
\left|H_{i}^{\prime}(M)\right| \leq \Lambda_{i}^{M}\left(\gamma_{i}, p_{i}, \rho_{i}, M\right) .
$$

In the case where $G \in \mathcal{G}_{\left(\xi_{1}, \xi_{2}\right) \cdot D}\left(\delta, g_{0}\right)$ or $G \in \mathcal{G}_{\left(\xi_{1}, \xi_{2}\right) \cdot D}\left(\delta, g_{0}, \varepsilon_{0}\right)$, we define the classes

$$
\mathcal{S}^{\xi_{1}, \xi_{2}}(\Omega), \quad \mathcal{S}_{\varepsilon_{0}}^{\xi_{1}, \xi_{2}}(\Omega), \quad \mathcal{S}_{\varepsilon_{0}}^{\xi_{1}, \xi_{2}}(\Omega, M), \quad \mathcal{S}_{\varepsilon_{0}}^{\xi_{1}, \xi_{2}}\left(\Omega, M, \Lambda_{i}^{M}\right)
$$

Similar definitions holds for $\beta$-Hölder control, and Lipschitz control. We pointed out that $\|u\|_{L^{\infty}(\Omega)} \leq M$ is a natural condition because of the Theorem 4.1. We also observe that the condition (2.3) is not artificial. In fact, if we change the condition (HC) to

$$
0 \leq H_{i}^{\prime}(t) \leq \gamma_{i} \cdot \max \left\{t^{p_{i}}, t^{\rho_{i}}\right\},
$$

both conditions (HC) and (2.3) are satisfied.

We are ready to present our main results. Suppose initially that $q>\frac{1+\delta}{\delta-\rho^{*}}$, where $\rho^{*}=$ $\max \left\{\rho_{1}, \rho_{2}\right\}$.

Theorem 2.1 Let $u \in \mathcal{S}_{\varepsilon_{0}}\left(\Omega, M, \Lambda_{i}^{M}\right)$. Assume that $\delta \geq 1$ and $q \geq n$. Then, $\nabla u \in B M O_{\text {loc }}(\Omega)$. More precisely, for any $\Omega^{\prime} \Subset \Omega$ there exists a constant $C=$ $C\left(n, \delta, g_{0}, q, \gamma_{i}, \lambda_{i}, \mu_{i}, M, \Lambda_{i}^{M},|\Omega|\right)>0$ such that

$$
\|\nabla u\|_{B M O\left(\Omega^{\prime}\right)} \leq \frac{C}{\varepsilon_{0}}\left(\operatorname{dist}\left(\Omega^{\prime}, \partial \Omega\right)^{-n}+\operatorname{diam}(\Omega)^{n} \cdot \varepsilon_{0}^{-1 / \delta}\right)^{\frac{1}{1+\delta}} .
$$

Moreover, $u$ is locally Log-Lipschitz and the following estimate holds

$$
|u(x)-u(y)| \leq\left. C(n)|| \nabla u\right|_{B M O\left(\Omega^{\prime}\right)}|x-y||\log | x-y|| .
$$

In particular, $u \in C_{\text {loc }}^{0, \alpha}(\Omega)$ for all $\alpha \in(0,1)$.

For the next results, we consider $\Theta_{u}^{-}$and $\Theta_{u}^{+}$the density functions of the negative and positive sets along the free boundaries for $u$, that is:

$$
\Theta_{u}^{-}\left(x_{0}, r\right)=\frac{\left|\{u<0\} \cap B_{r}\left(x_{0}\right)\right|}{\left|B_{r}\left(x_{0}\right)\right|} \quad \text { and } \quad \Theta_{u}^{+}\left(x_{0}, r\right)=\frac{\left|\{u>0\} \cap B_{r}\left(x_{0}\right)\right|}{\left|B_{r}\left(x_{0}\right)\right|}
$$

where $x_{0} \in F^{ \pm}(u)=F^{+}(u) \cup F^{-}(u):=(\partial\{u>0\} \cap \Omega) \cup(\partial\{u<0\} \cap \Omega)$.

Theorem 2.2 Assume that $q=\infty$. There exists a constant $c_{0}=c_{0}\left(n, \delta, g_{0}, \varepsilon_{0}, p_{i}, \rho_{i}, \gamma_{i}\right.$, $\left.\lambda_{i}, \mu_{i}, M, \xi_{i}\right) \in(0,1)$ such that the estimate

$$
|u(x)| \leq \frac{2 M}{c_{0}} \cdot\left|x-x_{0}\right|, \quad \forall x \in B_{1}\left(x_{0}\right)
$$

holds for any $u \in S_{\varepsilon_{0}}^{\xi_{1}, \xi_{2}}\left(B_{1}\left(x_{0}\right), M\right)$ provided

$$
\min \left\{\Theta_{u}^{+}\left(x_{0}, r\right), \Theta_{u}^{-}\left(x_{0}, r\right)\right\} \leq c_{0}, \quad \forall r \in(0,1) .
$$


Corollary 2.1 Assume that $q=\infty$. There exist positive constants $c_{0}^{*}=c_{0}^{*}\left(n, \delta, g_{0}\right.$, $\left.p_{i}, \rho_{i}, \gamma_{i}, M, \xi_{i}\right)$ and $r_{0}^{*}=r_{0}^{*}\left(\delta, g_{0}, \varepsilon_{0}, \lambda_{i}, \mu_{i}\right)$ such that the estimate

$$
|u(x)| \leq \frac{2 M}{c_{0}^{*} \cdot r_{0}} \cdot\left|x-x_{0}\right|, \quad \forall x \in B_{r_{0}}\left(x_{0}\right)
$$

holds for any $u \in S_{\varepsilon_{0}}^{\xi_{1}, \xi_{2}}\left(B_{r_{0}}\left(x_{0}\right), M\right)$ provided $0<r_{0} \leq r_{0}^{*}$ and

$$
\min \left\{\Theta_{u}^{+}\left(x_{0}, r\right), \Theta_{u}^{-}\left(x_{0}, r\right)\right\} \leq c_{0}^{*}, \quad \forall r \in\left(0, r_{0}\right) .
$$

Theorem 2.3 Let $u \in S_{\varepsilon_{0}}^{\xi_{1}, \xi_{2}}\left(\Omega, M, \Lambda_{i}^{M}\right)$ with $q=\infty$ and $\sigma \in(0,1]$. Assume that for any

$$
x_{0} \in\left\{x \in F^{ \pm}(u): \max \left\{\operatorname{dist}\left(x, F^{+}(u)\right), \operatorname{dist}\left(x, F^{-}(u)\right)\right\}<\sigma \cdot r_{0}^{*}\right\}
$$

the following condition is satisfied

$$
\min \left\{\Theta_{u}^{-}\left(x_{0}, r\right), \Theta_{u}^{+}\left(x_{0}, r\right)\right\} \leq c_{0}^{*},
$$

for all $0<r<\min \left\{\sigma \cdot r_{0}^{*}\right.$, dist $\left.\left(x_{0}, \partial \Omega\right)\right\}$, where $c_{0}^{*}$ and $r_{0}^{*}$ are as in Corollary 2.1. Then, $u \in C_{\text {loc }}^{0,1}(\Omega)$. More precisely, for any $\Omega^{\prime} \Subset \Omega$ there exists $C=$ $C\left(n, \delta, g_{0}, \varepsilon_{0}, p_{i}, \rho_{i}, \gamma_{i}, \lambda_{i}, \mu_{i}, M, \Lambda_{i}^{M}, \xi_{i}, \operatorname{diam}(\Omega)\right)>0$ such that, for $r_{\sigma}^{*}:=\min \{\sigma$. $\left.r_{0}^{*}, \operatorname{dist}\left(\Omega^{\prime}, \partial \Omega\right)\right\}$,

$$
\|\nabla u\|_{L^{\infty}\left(\Omega^{\prime}\right)} \leq \frac{C}{r_{\sigma}^{*}}
$$

At last, consider the following notation:

$$
\begin{aligned}
{[u]_{C^{0,1}\left(B_{\rho}(x)\right)}(x) } & =\sup _{y \in B_{\rho}(x), y \neq x} \frac{|u(y)-u(x)|}{|y-x|}, \\
{[u]_{C^{0,1}\left(B_{\rho}(x)\right)} } & =\sup _{z, w \in B_{\rho}(x), z \neq w} \frac{|u(z)-u(w)|}{|z-w|} .
\end{aligned}
$$

Theorem 2.4 Let $u \in S_{\varepsilon_{0}}^{\xi_{1}, \xi_{2}}(\Omega, M)$ and $\Omega^{\prime} \Subset \Omega$. Assume that $q=\infty$. Consider the sets

$$
\mathcal{S}_{p}\left(\Omega^{\prime}\right):=\left\{x \in \overline{\Omega^{\prime}}:[u]_{C^{0,1}\left(B_{r}(x)\right)}(x)=\infty \text { forallo }<r<\operatorname{dist}\left(\Omega^{\prime}, \partial \Omega\right)\right\}
$$

and

$$
\mathcal{S}_{l}\left(\Omega^{\prime}\right):=\left\{x \in \overline{\Omega^{\prime}}:[u]_{C^{0,1}\left(B_{r}(x)\right)}=\infty \text { forall0 }<r<\operatorname{dist}\left(\Omega^{\prime}, \partial \Omega\right)\right\} .
$$

Then, $\mathcal{S}_{p}\left(\Omega^{\prime}\right) \subset \mathcal{S}_{l}\left(\Omega^{\prime}\right) \subset F^{+}(u) \cap F^{-}(u)$ and, for $d=\operatorname{dist}\left(\Omega^{\prime}, \partial \Omega\right)$,

$$
\limsup _{F^{ \pm}(u) \times(0, d) \ni(x, r) \rightarrow\left(x_{0}, 0\right)}\left(\min \left\{\Theta_{u}^{+}(x, r), \Theta_{u}^{-}(x, r)\right\}\right) \geq c_{0}^{*}, \quad \text { for every } x_{0} \in \mathcal{S}_{l}\left(\Omega^{\prime}\right) .
$$

Still,

$$
\limsup _{r \rightarrow 0}\left(\min \left\{\Theta_{u}^{+}\left(x_{0}, r\right), \Theta_{u}^{-}\left(x_{0}, r\right)\right\}\right) \geq c_{0}^{*}, \quad \text { for every } x_{0} \in \mathcal{S}_{p}\left(\Omega^{\prime}\right) .
$$

Here $c_{0}^{*}$ is as in Corollary 2.1 . 


\section{Preliminary results}

In this section, we present some background results that will be used throughout the paper. They are part of the theory of Orlicz-Sobolev spaces and of the regularity theory of singular/degenerate elliptic equations of the type $\mathcal{L}_{g} u=B(x, u, \nabla u)$. Some proofs can be found in $[6,17,18]$. Here, we use freely the definitions, results and properties of the $N$-functions obtained in Section 2 of [18].

Initially, we observe that the conditions (PC) and (QC) imply the properties below.

Lemma 3.1 Let $G \in \mathcal{G}\left(\delta, g_{0}\right)$. Then, for all $t, s>0$ :

$$
\begin{aligned}
& (g-1) \min \left\{s^{\delta}, s^{g_{0}}\right\} g(t) \leq g(s t) \leq \max \left\{s^{\delta}, s^{g_{0}}\right\} g(t) ; \\
& (g-2) \frac{\operatorname{tg}(t)}{1+g_{0}} \leq G(t) \leq \operatorname{tg}(t) ; \\
& (G-1) G \text { is convex and } C^{2}(0, \infty) ; \\
& (G-2) \frac{1}{1+g_{0}} \min \left\{s^{1+\delta}, s^{1+g_{0}}\right\} G(t) \leq G(s t) \leq\left(1+g_{0}\right) \max \left\{s^{1+\delta}, s^{1+g_{0}}\right\} G(t) ; \\
& (G-3) G(a+b) \leq 2^{g_{0}}\left(1+g_{0}\right)(G(a)+G(b)), \forall a, b>0 .
\end{aligned}
$$

Proof See Lemma 1.1 of [17], Lemma 2.1 and Remark 2.2 of [18].

The next lemma is useful to prove the $L^{\infty}$ estimates for minimizers (see Theorem 4.1).

Lemma 3.2 Let $u \in W^{1, p}(\Omega), 1<p \leq n$, such that $\sup _{\partial \Omega} u<\infty$. For $k \geq k_{0}>\sup _{\partial \Omega} u$ assume that

$$
\int_{\Omega_{k}}|\nabla u|^{p} \mathrm{~d} x \leq C \cdot k^{\alpha}\left|\Omega_{k}\right|^{1-\frac{p}{n}+\varepsilon}, \quad \Omega_{k}:=\{u>k\},
$$

where $C, \varepsilon>0$ and $0 \leq \alpha \leq p+\varepsilon$. Then, there exists a positive constant $C^{*}$ depending on $n, \alpha, p, C, k_{0}$ and $\|u\|_{L^{1}\left(\Omega_{k_{0}}\right)}$ such that

$$
\sup _{\Omega} u \leq C^{*} .
$$

Proof See Lemma 5.2, Chapter 2 in [12].

The following result is a version of the Morrey's Lemma in the Orlicz-Sobolev setting. This Lemma performs a fundamental role to obtain the $C^{0, \alpha}$ estimates for minimizers.

Lemma 3.3 (Morrey's type Lemma) Let $u \in W_{l o c}^{1,1}(\Omega) \cap L^{1}(\Omega), G \in \mathcal{G}\left(\delta, g_{0}\right)$ and $0<\alpha \leq$ 1. Suppose $\Omega^{\prime} \subset \subset \Omega$ such that

$$
f_{B_{r}\left(x_{0}\right)} G(|\nabla u|) \mathrm{d} x \leq L r^{\alpha-1} \text { for every } x_{0} \in \Omega^{\prime} \text { with } 0<r \leq R_{0} \leq \operatorname{dist}\left(\Omega^{\prime}, \partial \Omega\right) .
$$

Then, there exist $C_{1}=C_{1}\left(\alpha, n, g_{0}\right)>0$ and $C_{2}=C_{2}\left(\alpha, n, g_{0}, R_{0}\right)>0$ such that

$$
\begin{aligned}
& |u(x)-u(y)| \leq\left(C_{1} \cdot \max \left\{\frac{L}{G(1)}, 1\right\}\right) \cdot|x-y|^{\alpha}, \text { for } x, y \in \Omega^{\prime} \text { with }|x-y| \leq \frac{R_{0}}{2}, \\
& \|u\|_{L^{\infty}\left(\Omega^{\prime}\right)} \leq C_{2}\left(L+\|u\|_{L^{1}(\Omega)}\right), \\
& {[u]_{\alpha, \Omega^{\prime}} \leq \max \left(C_{1} \cdot \max \left\{\frac{L}{G(1)}, 1\right\}, \frac{2^{\alpha+1} \cdot\|u\|_{L^{\infty}\left(\Omega^{\prime}\right)}}{R_{0}^{\alpha}}\right) .}
\end{aligned}
$$


Proof See Appendix in [6].

We recall still that a function $v \in W^{1, G}(\Omega)$ is a weak solution for the equation $\mathcal{L}_{g} v=$ $B(x, v, \nabla v)$ in $\Omega$ if $v \in W^{1, G}(\Omega)$ and for all $\xi \in C_{0}^{\infty}(\Omega)$ we have

$$
-\int_{\Omega} g(|\nabla v|) \frac{\nabla v}{|\nabla v|} \nabla \xi \mathrm{d} x+\int_{\Omega} B(x, v, \nabla v) \xi \mathrm{d} x=0 .
$$

We state a version of Harnack inequality for $B(x, v, \nabla v):=f(x)$. The proof of this result can be found in [17] (see, for instance, Corollary 1.4 and Sections 3 and 4).

Lemma 3.4 (Harnack inequality) Let $G \in \mathcal{G}\left(\delta, g_{0}\right)$ and $u$ be a nonnegative weak solution to $\mathcal{L}_{g} u=f$ in $B_{r}$, where $g=G^{\prime}$ and $f \in L^{q}\left(B_{r}\right), q>n$. Then, there exists a constant $C=C\left(n, \delta, g_{0}, q\right)>0$ such that

$$
\sup _{B_{r / 2}} u \leq C\left(\inf _{B_{r / 2}} u+r g^{-1}\left(r^{1-\frac{n}{q}}\|f\|_{L^{q}\left(B_{r}\right)}\right)\right) .
$$

The next Theorem compiles some estimates on the regularity theory of weak solutions to elliptic equations in Orlicz context (see, for instance, Theorem 1.7 and Lemma 5.1 in [17]). We present a special version only with the desired estimates.

Theorem 3.1 Let $G \in \mathcal{G}\left(\delta, g_{0}\right)$ and $v$ be a weak solution to $\mathcal{L}_{g} v=f$ in $\Omega$, where $g=G^{\prime}$ and $f \in L^{q}(\Omega), q>n$. Then $v$ is $C_{l o c}^{1, \alpha}(\Omega)$ for some constant $\alpha\left(n, g_{0}, \delta, q\right) \in(0,1)$. Moreover, for any $\Omega^{\prime} \Subset \Omega$, there exists a constant $C_{0}>0$, depending only on $n, \delta, g_{0}, q$ and $\operatorname{dist}\left(\Omega^{\prime}, \partial \Omega\right)$ such that

$$
\|v\|_{C^{1, \alpha}\left(\Omega^{\prime}\right)} \leq C_{0}\left(\|v\|_{L^{\infty}(\Omega)}+g^{-1}\left(\|f\|_{L^{q}(\Omega)}\right)\right) .
$$

Also, there exists a constant $C_{1}=C_{1}\left(n, \delta, g_{0}, q\right)>0$ such that for all $B_{r} \subset \Omega$,

$$
\sup _{B_{r / 2}}|\nabla v| \leq C_{1}\left(\frac{\|v\|_{L^{\infty}\left(B_{r}\right)}}{r}+g^{-1}\left(r^{1-n / q}\|f\|_{L^{q}\left(B_{r}\right)}\right)\right) .
$$

Further, if $f \equiv 0$, for every $\epsilon \in(0, n)$, there exists $C_{2}=C_{2}\left(n, \epsilon, \delta, g_{0}\right)>0$ such that,

$$
\int_{B_{r / 2}} G(|\nabla v|) \mathrm{d} x \leq C_{2} \sup _{B_{r}}|v| \cdot r^{\epsilon} .
$$

Proof We prove (3.2). By standard covering procedure, it is sufficient to prove that

$$
\|v\|_{C^{1, \alpha}\left(B_{\frac{r}{2}}\right)}^{*} \leq C\left(\|v\|_{L^{\infty}\left(B_{r}\right)}+r g\left(r^{1-n / q}\|f\|_{L^{q}\left(B_{r}\right)}\right)\right),
$$

where $\|v\|_{C^{1, \alpha}\left(B_{\left.\frac{r}{2}\right)}\right)}^{*}:=\|v\|_{L^{\infty}\left(B_{\frac{r}{2}}\right)}+r\|\nabla v\|_{L^{\infty}\left(B_{\frac{r}{2}}\right)}+r^{1+\alpha}[\nabla v]_{C^{0, \alpha}\left(B_{\frac{r}{2}}\right)}$ and $[\nabla v]_{C^{0, \alpha}\left(B_{\left.\frac{r}{2}\right)}^{+}\right)}$ is the semi-norm Hölder of $\nabla v$. By scaling of the type $v_{r}(x)=v(r x) / r$, we can assume that $r=1$. Now, for $\varepsilon>0$, consider

$$
\kappa_{0}:=\|u\|_{L^{\infty}\left(B_{1}\right)}+g^{-1}\left(\|f\|_{L^{q}\left(B_{1}\right)}\right)+\varepsilon \text { and } w(x):=\kappa_{0}^{-1} \cdot v(x), \quad \text { for } \quad x \in B_{1} .
$$

This way, it is easy to check that $\mathcal{L}_{g^{*}} w=\frac{f(x)}{g\left(\kappa_{0}\right)}$ in $B_{1}$ in the distributional sense, where $g^{*}(t)=$ $\frac{g\left(\kappa_{0} \cdot t\right)}{g\left(\kappa_{0}\right)}$. Now, since $g^{*}$ satisfies $(\mathrm{PC})$ and $(\mathrm{QC}), g^{*}(1)=1,\|w\|_{L^{\infty}\left(B_{1}\right)} \leq 1$ and $\left\|f_{0}\right\|_{L^{q}\left(B_{1}\right)} \leq$ 
1, we have by Theorem 1.7 in [17] (see still comments in pages 347-350) that, for some $\alpha=\alpha\left(n, \delta, g_{0}, q\right) \in(0,1)$ and $C=C\left(n, \delta, g_{0}, q\right)>0$,

$$
w \in C^{1, \alpha}\left(B_{1 / 2}\right) \quad \text { and } \quad \kappa_{0}^{-1} \cdot\|v\|_{C^{1, \alpha}\left(B_{1 / 2}\right)}=\|w\|_{C^{1, \alpha}\left(B_{1 / 2}\right)} \leq C .
$$

This implies the desired estimate taking $\varepsilon \rightarrow 0$. The proof of (3.3) follows immediately by (3.2), and (3.4) can be founded in Lemma 5.1 [17] (see also Remark 3.2 in [6]).

\section{Existence theory and boundedness for minimizers of $\mathcal{J}_{G}$.}

In this section, we establish existence and boundedness of (global) minimizers for the functional $\mathcal{J}_{G}$. The procedure to determine the existence of minimizers is standard, so we only present some steps. Before, we need of a definition.

Definition 4.1 We say $u \in W^{1, G}(\Omega)$ is a minimizer for $\mathcal{J}_{G}$ over $K_{\varphi}$ if

$$
\mathcal{J}_{G}(u, \Omega)=\min _{v \in K_{\varphi}} \mathcal{J}_{G}(v, \Omega),
$$

where for $\varphi \in W^{1, G}(\Omega) \cap L^{\infty}(\partial \Omega)$ with $\int_{\Omega} G(\nabla \varphi \mid) \mathrm{d} x<\infty$ and

$$
K_{\varphi}=\left\{v \in L^{1}(\Omega): \int_{\Omega} G(|\nabla v|) \mathrm{d} x<\infty, \quad v=\varphi \text { on } \partial \Omega\right\} .
$$

Minimizers over $K_{\varphi}$ are also called global minimizers.

In order to make shorter the mathematical estimates in this section, we consider the following simplifications

$$
[v]_{G}:=\int_{\Omega} G(|v|) \mathrm{d} x \text { and }[v]_{p}:=\|v\|_{L^{p}(\Omega)},
$$

for any $N$-function $G$ and $1 \leq p \leq \infty$.

Proposition 4.1 Let $G \in \mathcal{G}\left(\delta, g_{0}\right)$ and $\mathcal{J}_{G}(\cdot, \Omega)$ a functional of the type (2.2). Assume that $H(|\varphi|) \in L^{q^{\prime}}(\Omega)$ where $1 / q+1 / q^{\prime}=1$. Then, there exists, at least, one (global) minimizer for the optimization problem

$$
\min _{v \in K_{\varphi}} \mathcal{J}_{G}(v, \Omega)
$$

Remark 4.1 In the proof of the above proposition we need that for any $\psi \in W^{1, G}(\Omega)$ the condition $H_{i}(|\psi|)^{q^{\prime}} \in L^{G}(\Omega)$ to be satisfied. This condition is always true if $\delta>n-1$. When $0<\delta \leq n-1$, it is sufficient to assume that $\left(1+\rho_{i}\right) q^{\prime}<1+\delta, i=1,2$. We observe that this happens if we impose that $\delta>\rho^{*}=\max \left\{\rho_{1}, \rho_{2}\right\}$ and $q>\frac{1+\delta}{\delta-\rho^{*}}$. From now on, we assume that this conditions holds.

Proof of Proposition 4.1 First we note that

$$
\min _{v \in K_{\varphi}} \mathcal{J}_{G}(v, \Omega) \leq[\nabla \varphi]_{G}+\sum\left(\left[f_{i}\right]_{q}\left[H_{i}(|\varphi|)\right]_{q^{\prime}}+\left[h_{i}\right]_{\infty}|\Omega|\right) .
$$

Now, we claim that there exists $C=C(\varphi)>0$ such that $\mathcal{J}_{G}(v, \Omega) \geq-C$ for any $v \in K_{\varphi}$. In fact, by Poincaré type inequality (Lemma 2.4 of [18]), (G - 2) and (G - 3)

$C\left(g_{0}, d\right)[v-\varphi]_{G} \leq[\nabla v-\nabla \varphi]_{G} \leq C\left(g_{0}\right)\left([\nabla v]+[\nabla \varphi]_{G}\right)$ and $c\left(g_{0}\right)[v]_{G}-[\varphi]_{G} \leq[v-\varphi]_{G}$, 
where $d=\operatorname{diam}(\Omega)$. Combination of the inequalities in (4.2) give us

$$
-C\left(g_{0}, d\right)\left([\varphi]_{G}+[\nabla \varphi]_{G}\right) \leq[\nabla v]_{G}-c[v]_{G},
$$

where $c=c\left(g_{0}, d\right)<1$. For $P_{i}(t)=t^{\left(1+\rho_{i}\right) q^{\prime}}$ we have that $G \circ P_{i}^{-1}$ is a $N$-function. Thus, for $0<\kappa<1$, follows by $(\mathrm{G}-2)$ together with Young's inequality that,

$$
\left[f_{1} H_{1}\left(v^{+}\right)\right]_{1} \leq C_{1} \cdot[f]_{q}\left(\kappa^{-\left(1+\rho_{1}\right)}[\kappa v]_{\left(1+\rho_{1}\right) q^{\prime}}^{1+\rho_{1}}+|\Omega|^{1 / q^{\prime}}\right) \leq \frac{c}{4}[v]_{G}+\widetilde{C}_{1},
$$

were $C_{1}=2^{1+\rho_{1}+1 / q^{\prime}} \gamma_{1}$ and $\kappa=\left[4\left(1+g_{0}\right) c^{-1}\right]^{-\frac{1}{(1+\delta) q^{\prime}}}$. Similarly,

$$
\left[f_{2} H_{2}\left(v^{-}\right)\right]_{1} \leq \frac{c}{4}[v]_{G}+\widetilde{C}_{2} .
$$

Combining the estimates (4.3), (4.4) and (4.5) the claim is proved. Now, the proof follows similarly as in Theorem 3.1 of [18] considering a minimizing sequence $\left\{u_{j}\right\} \subset K_{\varphi}$ for $\mathcal{J}_{G}(v, \Omega)$.

Remark 4.2 It is possible to show the existence of minima if $q=\frac{1+\delta}{\delta-\rho^{*}}$ provided $[f]_{q}$ is small enough.

The next Lemma provides a universal control on the $L^{1}$-norm of minima. We combine these estimate with Lemma 3.2 to obtain $L^{\infty}$ estimates for minimizers.

Lemma 4.1 ( $L^{1}$-estimate) Let $u$ a minimizer for $\mathcal{J}_{G}$ in $K_{\varphi}$. Then,

$$
[u]_{1} \leq C=C\left(n, \delta, g_{0}, q, f_{i}, h_{i}, \gamma_{i}, \rho, \varphi, \Omega\right) .
$$

Proof By Young and Poincaré type inequalities, $(G-2)$ and $(G-3)$

$$
[u]_{1} \leq[u]_{G}+\widetilde{G}(1)|\Omega| \text { and }[u]_{G} \leq C\left(\delta, g_{0}, d\right)\left([\nabla u]_{G}+[\varphi]_{G}+[\nabla \varphi]_{G}\right),
$$

where $\widetilde{G}$ is the $N$-complementary function of $G$ and $d=\operatorname{diam}(\Omega)$. By minimality, we have

$$
[\nabla u]_{G} \leq \mathcal{J}_{G}(\varphi)+\left[f_{1} H_{1}\left(u_{j}{ }^{+}\right)\right]_{1}+\left[f_{2} H_{2}\left(u_{j}{ }^{-}\right)\right]_{1}+C_{1} .
$$

Similarly as in the proof of the Proposition 4.1, we obtain that $[\nabla u]_{G} \leq C$ and by (4.6) the proof is now complete.

Below we establish the $L^{\infty}$ estimates for (global) minimizers.

Theorem 4.1 ( $L^{\infty}$-estimate) Let $G \in \mathcal{G}\left(\delta, g_{0}\right), \mathcal{J}_{G}$ a functional as in (2.2) and $u$ be a minimizer for $\mathcal{J}_{G}$ in $K_{\varphi}$. Then, $u \in L^{\infty}(\Omega)$. In fact,

$$
[u]_{\infty} \leq C=C\left(n, \delta, g_{0}, q, f_{i}, h_{i}, \gamma_{i}, \rho_{i}, \varphi, \Omega\right) .
$$

Proof We prove upper boundedness of $u$. If $\delta>n-1$, since $W^{1,1+\delta}(\Omega) \hookrightarrow W^{1, G}(\Omega)$, the existence of the universal constant $C$ is an immediate consequence of the Rellich-Kondrachov embedding Theorem. Thus, we can assume that $0<\delta \leq n-1$. Let $k_{0}=\min \{k \in \mathbb{N}: k \geq$ $\left.\sup _{\partial \Omega} \varphi\right\}$. For each $k \geq k_{0}$, we define a truncation function $u_{k}: \Omega \longrightarrow \mathbb{R}^{n}$ by

$$
u_{k}= \begin{cases}u, & \text { if } u \leq k \\ k, & \text { if } u>k\end{cases}
$$

Obviously $u_{k} \in K_{\varphi}$ and, up to sets of measure zero,

$$
\left\{u_{k}>0\right\}=\{u>0\}, \quad\left\{u_{k}<0\right\}=\{u<0\}, \quad\left\{u_{k}=0\right\}=\{u=0\} .
$$


Setting $\Omega_{k}:=\{u>k\}$ we get by definition of $u_{k}$, minimality of $u,(\mathrm{HC}),(\mathrm{G}-3)$ and Hölder inequality that

$$
[\nabla u]_{G} \leq C_{1}\left[[(u-k)]_{\left(1+\rho_{1}\right) q^{\prime}}^{1+\rho_{1}}+k^{1+\rho_{1}}\left|\Omega_{k}\right|^{1 / q^{\prime}}\right] .
$$

Now, for any $\left(1+\rho_{1}\right) q^{\prime}<\delta_{0}<1+\delta$ we have by Sobolev's embedding and Theorem 2.2 in [18] that $u \in W^{1, \delta_{0}}(\Omega)$. So, applying Hölder, Gagliardo-Nirenberg-Sobolev and Young inequalities in the left side in the above estimate we get

$$
[(u-k)]_{\left(1+\rho_{1}\right) q^{\prime}}^{1+\rho_{1}} \leq C_{2}\left|\Omega_{k}\right|^{(1-\widetilde{\beta}) / q^{\prime}} \cdot[\nabla u]_{\delta_{0}}^{1+\rho_{1}} \leq C_{3}\left|\Omega_{k}\right|^{1 / q^{\prime}}+\frac{1}{2 C_{1}} \cdot[\nabla u]_{\delta_{0}}^{\delta_{0}},
$$

where $\widetilde{\beta}=\frac{\left(1+\rho_{1}\right)\left(n-\delta_{0}\right)}{n \delta_{0}}$. Hence, combining (4.9) and (4.10), and one more time by Young inequality, we obtain

$$
[\nabla u]_{\delta_{0}}^{\delta_{0}}-\widetilde{G}_{\delta_{0}}(1)|\Omega|^{1 / q}\left|\Omega_{k}\right|^{1 / q^{\prime}} \leq \frac{1}{2}[\nabla u]_{\delta_{0}}^{\delta_{0}}+C_{4} k^{1+\rho_{1}}\left|\Omega_{k}\right|^{1 / q^{\prime}},
$$

where $\widetilde{G}_{\delta_{0}}$ is the $N$-complementary function of $G \circ P^{-1}$, with $P(t)=t^{\delta_{0}}$. By (4.11) we conclude that

$$
\int_{\Omega_{k}}|\nabla u|^{\delta_{0}} \mathrm{~d} x \leq C_{5} \cdot k^{1+\rho_{1}}\left|\Omega_{k}\right|^{1 / q^{\prime}}=C_{5} k^{1+\rho_{1}}\left|\Omega_{k}\right|^{1-\frac{\delta_{0}}{n}+\varepsilon},
$$

where $\varepsilon=\frac{1}{q^{\prime}}+\frac{\delta_{0}}{n}-1$. Since $\sup _{\partial \Omega} u \leq[\varphi]_{\infty}$ the upper boundedness of $u$ is consequence of Lemma 4.1 together with Lemma 3.2. By similar procedure we prove that $\inf _{\Omega} u \geq-C$. This completes the proof.

Remark 4.3 An immediate consequence of the Theorem above is a uniform control of the $W^{1, G}$-norm of the minimizers depending only on universal constants and intrinsic parameters.

Remark 4.4 If $\varphi \geq 0$ and $f_{2} \geq 0$, then $u \geq 0$. In particular $u$ minimizes the functional,

$$
E_{G}(v, \Omega)=\int_{\Omega}\left[G(|\nabla v|)+f_{1}(x) H_{1}\left(v^{+}\right)+h_{1}(x) \chi_{\{v>0\}}\right] \mathrm{d} x .
$$

In fact, for $\widetilde{Q}\left(h_{1}, h_{2}\right)(v)=\left(h_{1}-h_{2}\right) \chi_{\{v>0\}}-\left(h_{1}-h_{2}\right)^{-} \chi_{\{v=0\}}$, we observe that $u$ minimize

$$
\widetilde{E}_{G}(v):=\int_{\Omega}\left[G(|\nabla u|)+f_{1}(x) H_{1}\left(u^{+}\right)+f_{2}(x) H_{2}\left(u^{-}\right)+\widetilde{Q}\left(h_{1}, h_{2}\right)(u)(x)\right] \mathrm{d} x .
$$

Now, let $u_{\varepsilon}(x)=u(x)+\varepsilon u^{-}$for $0<\varepsilon<1$. By the minimality of $u$ with respect to $\widetilde{E}_{G}$, we obtain

$$
\int_{\{u<0\}}\left\{G(|\nabla u|)+f_{2}\left[H_{2}\left(u^{-}\right)-H_{2}\left(u_{\varepsilon}^{-}\right)\right]\right\} \mathrm{d} x \leq\left(1+g_{0}\right)(1-\varepsilon)^{1+\delta} \int_{\{u<0\}} G(|\nabla u|) \mathrm{d} x .
$$

Since $H_{2}$ is increasing follows for $\varepsilon \ll 1$ and by boundary condition that $u \geq 0$.

\section{The uniform Hölder regularity for local minimizers in $\mathcal{S}_{\varepsilon_{0}}(\Omega, M)$}

For the purpose of the Hölder, log-Lipschitz and Lipschitz regularity for minimizers, we do not need to consider global minimizers, or else, functions for which the $G$-energy is finite in the whole domain. We can consider only local minimizers as in [10]. Follows the precise definition. 
Definition 5.1 A function $u \in W_{l o c}^{1, G}(\Omega)$ is said to be a local minimizer for the functional $\mathcal{J}_{G}(\cdot, \Omega)$ if for any $W^{1, G}$-perturbation compactly supported, i.e, $\psi \in W_{c}^{1, G}(\Omega)$, we have

$$
\mathcal{J}_{G}(u, \Omega) \leq \mathcal{J}_{G}(u+\psi, \Omega) .
$$

It follows that $u$ is a local minimizer for $\mathcal{J}_{G}(\cdot, \Omega)$ if and only if $\forall D \subset \subset \Omega$ with Lipschitz boundary

$$
\mathcal{J}_{G}(u, D)=\min _{v \in K_{u}} \mathcal{J}_{G}(v, D), \quad K_{u}=\left\{v \in W^{1, G}(D): v=u \text { on } \partial D\right\} .
$$

It is easy to check that global minimizers are also local minimizers. By simplicity, we call local minimizers only minimizers.

We now aim towards the uniform (local) Hölder regularity for minimizers in the class $\mathcal{S}_{\varepsilon_{0}}(\Omega, M)$. Here, we assume that $q>n$ if $\delta>n-1$ or $q>\max \left\{\frac{1+\delta}{\delta-\rho^{*}}, n\right\}$ when $0<\delta \leq$ $n-1$. The proof follows the same spirit of the proof of the Theorem 4.1 of [18] and Theorem 5.1 in [6]. As mentioned in [6], the non-degeneracy condition (bound from below on $G(1)$ ) is crucial for the uniform Hölder estimate.

Theorem 5.1 Let $u \in \mathcal{S}_{\varepsilon_{0}}(\Omega, M)$ and $\alpha \in\left(0,1-\frac{n}{q}\right)$. Then $u$ is uniformly in $C_{\text {loc }}^{0, \alpha}(\Omega)$. More precisely, for any $\Omega^{\prime} \Subset \Omega$, there exists a positive constant $\bar{C}=$ $\bar{C}\left(\delta, g_{0}, p_{i}, \rho_{i}, \gamma_{i}, \Lambda_{i}, \lambda_{i}, \mu_{i}, q, M\right)$ such that

$$
[u]_{C^{0, \alpha}\left(\Omega^{\prime}\right)} \leq C:=\max \left(C_{1} \cdot \max \left\{\frac{\bar{C}}{\varepsilon_{0}}, 1\right\}, \frac{2^{\alpha+1} \cdot M}{\min \left\{\left(\frac{1}{2}\right)^{1+1 / \varepsilon}, \operatorname{dist}\left(\Omega^{\prime}, \partial \Omega\right)\right\}^{\alpha}}\right),
$$

where $\varepsilon=\varepsilon(n, q, \alpha) \in(0,1)$ and $C_{1}=C_{1}\left(\delta, g_{0}, n\right)>0$. In fact,

$$
C=C\left(n, \delta, g_{0}, p_{i}, \rho_{i}, \gamma_{i}, \Lambda_{i}, \lambda_{i}, \mu_{i}, q, \varepsilon_{0}, M, \alpha, \operatorname{dist}\left(\Omega^{\prime}, \partial \Omega\right)\right) .
$$

Proof Since $u \in \mathcal{S}_{\varepsilon_{0}}(\Omega, M), u$ is a local minimizer of a functional of the type

$$
\mathcal{J}_{G}(v, \Omega)=\int_{\Omega}\left[G(|\nabla v|)++f_{1}(x) H_{1}\left(v^{+}\right)+f_{2}(x) H_{2}\left(v^{-}\right)+Q\left(h_{1}, h_{2}\right)(v)(x)\right] \mathrm{d} x
$$

satisfying the conditions of the Definition 2.3. Fix $\alpha \in(0,1-n / q), \Omega^{\prime} \Subset \Omega$ and $y \in \Omega^{\prime}$. Now, consider $r \in(0,1)$ such that $B_{r}(y) \subset \Omega$. By simplify the notation, assume $y=0$. Let $w$ be the solution to the Dirichlet problem

$$
\mathcal{L}_{g} w=0 \text { in } B_{r} \quad \text { and } \quad w-u \in W_{0}^{1, G}\left(B_{r}\right)
$$

From Theorem 2.3 of [18] we have

$$
\begin{aligned}
& \int_{B_{r}}[G(|\nabla u|)-G(|\nabla w|)] \mathrm{d} x \geq C_{1}\left(g_{0}, \delta\right)\left(\int_{A_{2}} G(|\nabla u-\nabla w|) \mathrm{d} x\right. \\
& \left.\quad+\int_{A_{1}} F(|\nabla u|)|\nabla u-\nabla w|^{2} \mathrm{~d} x\right),
\end{aligned}
$$

where

$$
A_{1}=\left\{x \in B_{r}:|\nabla u-\nabla w| \leq 2|\nabla u|\right\}, \quad A_{2}=\left\{x \in B_{r}:|\nabla u-\nabla w|>2|\nabla u|\right\}
$$


and $F(t)=g(t) / t$. On the other hand, by Maximum Principle (Lemma 2.8 of [18]) we have $\|w\|_{L^{\infty}\left(B_{r}\right)}=\|u\|_{L^{\infty}\left(B_{r}\right)} \leq M$. Hence, follows by the minimality of $u$ and Hölder inequality that

$$
\int_{B_{r}}[G(|\nabla u|)-G(|\nabla w|)] \mathrm{d} x \leq C_{2} \cdot r^{n / q^{\prime}},
$$

where $C_{2}=C_{2}\left(n, \lambda_{i}, \mu_{i}, \Lambda_{i}, \gamma_{i}, M,|\Omega|, q\right)>0$. Combination of (5.1) and (5.2) reveals that

$$
\max \left\{\int_{A_{2}} G(|\nabla u-\nabla w|) \mathrm{d} x, \int_{A_{1}} F(|\nabla u|)|\nabla u-\nabla w|^{2} \mathrm{~d} x\right\} \leq C_{3} \cdot r^{n / q^{\prime}} .
$$

Let $\varepsilon>0$ and suppose that $r^{\varepsilon} \leq 1 / 2$. By Hölder inequality, $(g-2)$ and (5.3) we get

$$
\int_{A_{1} \cap B_{r^{1+\varepsilon}}} G(|\nabla u-\nabla w|) \mathrm{d} x \leq C_{4} \cdot r^{n / 2}\left(\int_{B_{r^{1+\varepsilon}}} G(|\nabla u|) \mathrm{d} x\right)^{1 / 2} .
$$

Combining now (5.3) and (5.4) we arrive at

$$
\int_{B_{r^{1}+\varepsilon}} G(|\nabla u-\nabla w|) \mathrm{d} x \leq C_{5}\left[r^{n / q^{\prime}}+r^{n / 2}\left(\int_{B_{r^{1+\varepsilon}}} G(|\nabla u|) \mathrm{d} x\right)^{1 / 2}\right] .
$$

Now, it follows by (3.4) in Theorem 3.1, Maximum Principle and $(G-3)$ that for any $\bar{\beta} \in\left(0, n / q^{\prime}\right)$

$$
\int_{B_{r^{1}+\varepsilon}} G(|\nabla u|) \mathrm{d} x \leq C_{6}\left[r^{\bar{\beta}}+r^{\bar{\beta} / 2}\left(\int_{B_{r^{1}+\varepsilon}} G(|\nabla u|) \mathrm{d} x\right)^{1 / 2}\right],
$$

where $C_{6}=C_{6}\left(\delta, g_{0}, n, \lambda_{i}, \mu_{i}, \Lambda_{i}, \gamma_{i}, M,|\Omega|, q, \bar{\beta}\right)>0$. Hence as in Theorem 4.1 of [18], we obtain

$$
\int_{B_{r 1+\varepsilon}} G(|\nabla u|) \mathrm{d} x \leq\left[\left(C_{6}+1\right)^{1 / 2}+C_{6}^{1 / 2}\right]^{2} C_{6} \cdot r^{\bar{\beta}} .
$$

Taking $\bar{\beta}:=(1+\varepsilon)(n-(1-\alpha))$, we choose $\varepsilon>0$ so that $0<\bar{\beta}<n / q^{\prime}$. Finally, by setting $\varrho_{0}:=\min \left\{\left(\frac{1}{2}\right)^{1+1 / \varepsilon}, \operatorname{dist}\left(\Omega^{\prime}, \partial \Omega\right)\right\}$ and, for $0<\varrho<\varrho_{0}, r=\varrho^{1 /(1+\varepsilon)}$, we have that $r^{\varepsilon} \leq 1 / 2$ and therefore by (5.7),

$$
\int_{B_{\varrho}} G(|\nabla u|) \mathrm{d} x \leq \bar{C} \varrho^{n+\alpha-1} .
$$

By Lemma 3.3 the prove of Theorem is finished.

By above result, we have that $\{u \neq 0\}$ is an open set. The following Corollary establishes the Euler-Lagrange equations for which minimizers are weak (sub/super) solutions.

Corollary 5.1 Let u a global (local) minimizer for $\mathcal{J}_{G}$ in $K_{\varphi}$. Then

$$
\mathcal{L}_{g} u=f_{1}(x) H_{1}^{\prime}\left(u^{+}\right)+f_{2}(x) H_{2}^{\prime}\left(u^{-}\right) \text {in }\{u \neq 0\},
$$

in the weak distributional sense. If $h_{1} \geq h_{2}$, we have

$$
\mathcal{L}_{g} u \geq f_{1}(x) H_{1}^{\prime}(u) \chi_{\{u>0\}}+f_{2}(x) H_{2}^{\prime}(-u) \chi_{\{u<0\}} \quad \text { in } \Omega .
$$


Similarly, if $h_{2} \geq h_{1}$, then

$$
\mathcal{L}_{g} u \leq f_{1}(x) H_{1}^{\prime}(u) \chi_{\{u>0\}}+f_{2}(x) H_{2}^{\prime}(-u) \chi_{\{u<0\}} \quad \text { in } \Omega .
$$

(ii) In this case where $f_{2}, \varphi \geq 0$, we have

$$
\mathcal{L}_{g} u-f_{1}(x) H_{1}^{\prime}(u) \chi_{\{u>0\}} \geq 0 \quad \text { in } \Omega .
$$

Proof The prove follows standard procedure as in [11] or [18].

\section{Lipschitz regularity for one-phase minimizers in $\mathcal{S}_{\varepsilon_{0}}\left(\Omega, M, \Lambda_{i}^{M}\right)$}

In this section, we address the local Lipschitz continuity for one-phase minimizers. We observe that with the restriction of the sign of $u$, the sharp regularity of minima holds in general class $\mathcal{S}_{\varepsilon_{0}}\left(\Omega, M, \Lambda_{i}^{M}\right)$ when $q=\infty$. By Remark 4.4, the restriction of the sign of $u$ is obtained when we assume $f_{2}, \varphi \geq 0$. The proof this fact follows a combination of similar strategies founded in Theorem 3.1 of [16] and Lemma 4.2 of [18]. For the records, we state and prove this result.

Theorem 6.1 Let $0 \leq u \in \mathcal{S}_{\varepsilon_{0}}\left(\Omega, M, \Lambda_{i}^{M}\right)$. Suppose that $q=\infty$. Then, for any $\Omega^{\prime} \Subset \Omega$, there exists $C_{1}=C_{1}\left(n, \delta, g_{0}, q, \varepsilon_{0}, \gamma_{1}, \lambda_{1}, \mu_{1}, \rho_{1}, \Lambda_{1}^{M}, \Omega^{\prime}, \Omega\right)>0$ such that

$$
u(x) \leq \frac{C_{1}}{\operatorname{dist}\left(\Omega^{\prime}, \partial \Omega\right)} \cdot \operatorname{dist}(x, \partial\{u>0\}), \quad \forall x \in \Omega^{\prime} .
$$

In particular, $u \in C_{\text {loc }}^{0,1}(\Omega)$ and for some $C_{2}=C_{2}\left(C_{1}\right)>0$ holds the following estimate

$$
\|\nabla u\|_{L^{\infty}\left(\Omega^{\prime}\right)} \leq \frac{C_{2}}{\operatorname{dist}\left(\Omega^{\prime}, \partial \Omega\right)} .
$$

Proof Assume initially that $\operatorname{dist}\left(\Omega^{\prime}, \partial \Omega\right)=1$. Now, suppose by contradiction, that (6.1) does not hold. In this case, there exist a sequence of functions $u_{k} \in \mathcal{S}_{\varepsilon_{0}}\left(\Omega, M, \Lambda_{i}^{M}\right)$ and a sequence of points $x_{k} \in \Omega^{\prime} \cap\left\{u_{k}>0\right\}$ such that

$$
\frac{u_{k}\left(x_{k}\right)}{d_{k}} \geq k, \quad \text { where } \quad d_{k}:=\operatorname{dist}\left(x_{k}, \partial\left\{u_{k}>0\right\}\right) .
$$

Since $\left\{u_{k}\right\}$ is uniformly bounded we have $d_{k} \rightarrow 0$. Observe now that, for each $k \in \mathbb{N}$, we can found $y_{k} \in \partial\left\{u_{k}>0\right\}$ such that

$$
d_{k}=\left|x_{k}-y_{k}\right| .
$$

By definition of $\mathcal{S}_{\varepsilon_{0}}\left(\Omega, M, \Lambda_{i}^{M}\right)$, there exist $G_{k}, H_{k}, f_{k}$ and $h_{k}$ satisfying the conditions in Definition 2.3 such that $u_{k}$ is a minimizer of

$$
\mathcal{J}_{G_{k}}(u, \Omega)=\int_{\Omega}\left[G_{k}(|\nabla u|)+f_{k}(x) H_{k}\left(u^{+}\right)+h_{k}(x) \chi_{\{u>0\}}\right] \mathrm{d} x .
$$

Follows by Corollary 5.1 that

$$
\mathcal{L}_{g_{k}} u_{k}=f_{k} H_{k}^{\prime}\left(u_{k}\right) \text { in }\left\{u_{k}>0\right\},
$$

where $g_{k}=G_{k}^{\prime}$. Thus, by Harnack's inequality, we obtain

$$
u_{k}\left(x_{k}\right) \leq \sup _{\bar{B}_{\frac{3}{4} d_{k}}\left(x_{k}\right)} u_{k} \leq C_{1}\left(\inf _{\bar{B}_{\frac{3}{4} d_{k}}\left(x_{k}\right)} u_{k}+C_{2} \cdot d_{k}\right),
$$


where $C_{1}$ and $C_{2}$ depends on $n, \delta, g_{0}, \mu_{1}, \lambda_{1}, \rho_{1}, \gamma_{1}, M$ and $\Lambda_{1}^{M}$.

Since $\bar{B}_{\frac{3}{4} d_{k}}\left(x_{k}\right) \cap \bar{B}_{\frac{1}{4} d_{k}}\left(x_{k}\right) \neq \emptyset$ we conclude by (6.4) that

$$
\sup _{\bar{B}_{\frac{1}{4} d_{k}}\left(y_{k}\right)} u_{k} \geq c u_{k}\left(x_{k}\right)-C_{2} d_{k} .
$$

In sequel, consider the set

$$
\Omega_{k}:=\left\{z \in B_{d_{k}}\left(y_{k}\right): \operatorname{dist}\left(z, \partial\left\{u_{k}>0\right\}\right) \leq \frac{1}{3} \operatorname{dist}\left(z, \partial B_{d_{k}}\left(y_{k}\right)\right)\right\} .
$$

Since $y_{k} \in \partial\left\{u_{k}>0\right\}$, we have $B_{\frac{d_{k}}{4}}\left(y_{k}\right) \subseteq \Omega_{k}$ and hence

$$
\begin{aligned}
A_{k} & :=\sup _{z \in \Omega_{k}} \operatorname{dist}\left(z, \partial B_{d_{k}}\left(y_{k}\right)\right) \cdot u_{k}(z) \\
& \geq \inf _{B_{\frac{d_{k}}{4}}\left(y_{k}\right)} \operatorname{dist}\left(z, \partial B_{d_{k}}\left(y_{k}\right)\right) \cdot \sup _{B_{\frac{d_{k}}{4}}\left(y_{k}\right)} u_{k}(z) \\
& =\frac{3}{4} d_{k} \cdot \sup _{B_{\frac{d_{k}}{4}}\left(y_{k}\right)} u_{k}(z) .
\end{aligned}
$$

On the other hand, there exists $z_{k} \in \Omega_{k}$ such that

$$
A_{k}=\operatorname{dist}\left(z_{k}, \partial B_{d_{k}}\left(y_{k}\right)\right) \cdot u_{k}\left(z_{k}\right) .
$$

Combination of (6.6) and (6.7) lead us to conclude that

$$
u_{k}\left(z_{k}\right) \geq \frac{3}{4} \sup _{B_{\frac{d_{k}}{4}}\left(y_{k}\right)} u_{k}(z)
$$

and by (6.5) and (6.8) we arrive at

$$
u_{k}\left(z_{k}\right) \geq \frac{3}{4}\left(c u_{k}\left(x_{k}\right)-C_{2} d_{k}\right) .
$$

Let $w_{k} \in \partial\left\{u_{k}>0\right\}$ such that

$$
\left|z_{k}-w_{k}\right|=\operatorname{dist}\left(z_{k}, \partial\left\{u_{k}>0\right\}\right) .
$$

Now, as $z_{k} \in \Omega_{k}$ we get

$$
\delta_{k}:=\left|z_{k}-w_{k}\right| \leq \frac{1}{3}\left(d_{k}-\left|z_{k}-y_{k}\right|\right)
$$

and as $\delta_{k} \leq\left|z_{k}-y_{k}\right|$,

$$
\delta_{k} \leq \frac{1}{3}\left(d_{k}-\delta_{k}\right) \Longrightarrow \delta_{k} \leq \frac{1}{4} d_{k} .
$$

By above estimate and (6.9)

$$
\frac{u_{k}\left(z_{k}\right)}{\delta_{k}} \geq \frac{3}{4} \frac{4}{d_{k}}\left(c u_{k}\left(x_{k}\right)-C_{2} d_{k}\right)=3\left(c \cdot \frac{u_{k}\left(x_{k}\right)}{d_{k}}-C_{2}\right) .
$$

This implies, by (6.3), $\frac{u_{k}\left(z_{k}\right)}{\delta_{k}} \longrightarrow+\infty$. 
Once $\operatorname{dist}\left(x, \partial\left\{u_{k}>0\right\}\right) \leq \frac{\delta_{k}}{2}, \forall x \in B_{\frac{\delta_{k}}{2}}\left(w_{k}\right)$,

$$
\begin{aligned}
\operatorname{dist}\left(x, \partial B_{d_{k}}\left(y_{k}\right)\right) & \geq \operatorname{dist}\left(z_{k}, \partial B_{d_{k}}\left(y_{k}\right)\right)-\left|z_{k}-x\right| \\
& \geq \operatorname{dist}\left(z_{k}, \partial B_{d_{k}}\left(y_{k}\right)\right)-\left(\left|z_{k}-w_{k}\right|+\left|x-w_{k}\right|\right) \\
& \geq \operatorname{dist}\left(z_{k}, \partial B_{d_{k}}\left(y_{k}\right)\right)-\frac{3}{2} \delta_{k} \\
& \geq \frac{1}{2} \operatorname{dist}\left(z_{k}, \partial B_{d_{k}}\left(y_{k}\right)\right),
\end{aligned}
$$

and hence

$$
\operatorname{dist}\left(x, \partial\left\{u_{k}>0\right\}\right) \leq \frac{1}{6} \operatorname{dist}\left(z_{k}, \partial B_{d_{k}}\left(y_{k}\right)\right) \leq \frac{1}{3} \operatorname{dist}\left(x, \partial B_{d_{k}}\left(y_{k}\right)\right),
$$

i.e. $B_{\frac{\delta_{k}}{2}}\left(w_{k}\right) \subseteq \Omega_{k}$. Also, by estimate (6.12), we have that

$$
u_{k}\left(z_{k}\right)=\frac{A_{k}}{\operatorname{dist}\left(z_{k}, \partial B_{d_{k}}\left(y_{k}\right)\right)} \geq \frac{\operatorname{dist}\left(x, \partial B_{d_{k}}\left(y_{k}\right)\right) \cdot u_{k}(x)}{\operatorname{dist}\left(z_{k}, \partial B_{d_{k}}\left(y_{k}\right)\right)} \geq \frac{1}{2} u_{k}(x), \quad \forall x \in B_{\frac{\delta_{k}}{2}}\left(w_{k}\right) .
$$

Thus,

$$
\sup _{B_{\frac{\delta_{k}}{2}}\left(w_{k}\right)} u_{k} \leq 2 u_{k}\left(z_{k}\right)
$$

As $B_{\delta_{k}}\left(z_{k}\right) \subset\{u>0\}$, Harnack inequality secures that

$$
\inf _{\bar{B}_{\frac{3}{4} \delta_{k}}\left(z_{k}\right)} u_{k} \geq \widetilde{c} u_{k}\left(z_{k}\right)-\widetilde{C} \delta_{k} .
$$

Thus,

$$
\sup _{\bar{B}_{\frac{1}{4} \delta_{k}}\left(w_{k}\right)} u_{k} \geq \widetilde{c} u_{k}\left(z_{k}\right)-\widetilde{C} \delta_{k} .
$$

Let now $\xi_{k}: B_{1} \longrightarrow \mathbb{R}$ the following family of auxiliary functions:

$$
\xi_{k}(x)=\frac{u_{k}\left(w_{k}+\frac{\delta_{k}}{2} x\right)}{u_{k}\left(z_{k}\right)}, \quad x \in B_{1} .
$$

Clearly $\xi_{k}(0)=0$ and, by (6.13) and (6.14), for $k>>1$,

$$
\max _{B_{1}} \xi_{k} \leq 2 \quad \text { and } \quad \max _{B_{\frac{1}{2}}} \xi_{k} \geq \frac{3 \widetilde{c}}{4}>0 .
$$

Define still the following family of $\mathrm{N}$-functions

$$
\bar{G}_{k}(t):=\frac{G_{k}\left(\sigma_{k} t\right)}{\sigma_{k} g_{k}\left(\sigma_{k}\right)},
$$

where $\sigma_{k}=\frac{2 u_{k}\left(z_{k}\right)}{\delta_{k}}$. Observe that, for $k$ sufficient large, $\sigma_{k}>c \cdot k \longrightarrow+\infty$. By Proposition 6.1 of [6] we know that $\left\{\bar{G}_{k}\right\}_{k \geq 1} \subset \mathcal{G}\left(\delta, g_{0},\left(1+g_{0}\right)^{-1}\right)$ and $\bar{G}_{k} \leq 1$. Also, by simple computation, we observe that $\xi_{k}$ is a local minimizer of

$$
\mathcal{F}_{k}(\psi)=\int_{B_{1}}\left[\bar{G}_{k}(|\nabla \psi|)+\bar{f}_{k} \bar{H}_{k}\left(\psi^{+}\right)+\bar{h}_{k} \chi_{\{\psi>0\}}\right] \mathrm{d} x,
$$


where

$$
\bar{H}_{k}(v)=H_{k}\left(u_{k}\left(z_{k}\right) \cdot v\right), \quad \bar{f}_{k}(x)=\frac{f_{k}\left(w_{k}+\frac{\delta_{k}}{2} x\right)}{\sigma_{k} g_{k}\left(\sigma_{k}\right)}, \quad \bar{h}_{k}(x)=\frac{h_{k}\left(w_{k}+\frac{\delta_{k}}{2} x\right)}{\sigma_{k} g_{k}\left(\sigma_{k}\right)} .
$$

Now, we estimate

$$
\begin{aligned}
& \left\|\bar{h}_{k}\right\|_{L^{\infty}\left(B_{1}\right)} \leq C \cdot \frac{1+g_{0}}{\varepsilon_{0}} \cdot \frac{\mu_{1}}{k^{1+\delta}} \rightarrow 0 \quad \text { as } j \rightarrow \infty, \\
& \left\|\bar{f}_{k}(x) \bar{H}_{k}(v)\right\|_{L^{\infty}\left(B_{1}\right)} \leq C \cdot \frac{1+g_{0}}{\varepsilon_{0}} \cdot \frac{\lambda_{1}}{k^{1+\delta}} \cdot \gamma_{1}\left(1+M \cdot\|v\|_{L^{\infty}\left(B_{1}\right)}\right)^{1+\rho_{1}} \rightarrow 0,
\end{aligned}
$$

for any bounded function $v$.

Let us take $v_{k} \in W^{1, G}\left(B_{5 / 8}\right)$ such that

$$
\mathcal{L}_{k} v_{k}=0 \quad \text { in } \quad B_{5 / 8} \quad \text { and } \quad v_{k}=\xi_{k} \quad \text { in } \quad \partial B_{5 / 8} \text {, }
$$

where $\mathcal{L}_{k}$ is the operator related to $\bar{G}_{k}$.

By stability estimates (5.5) and (5.7) in Theorem 5.1, for $\varepsilon=0$ and $r=\frac{5}{8}$, Maximum Principle, (6.17) and (6.18) we obtain,

$$
\int_{B_{\frac{5}{8}}} \bar{G}_{k}\left(\left|\nabla v_{k}-\nabla \xi_{k}\right|\right) \mathrm{d} x \leq \widetilde{C} \cdot k^{-\frac{1+\delta}{2}}
$$

Since, for each $k$, holds

$$
\frac{1}{\left(g_{0}+1\right)^{2}} \min \left\{t^{1+\delta}, t^{1+g_{0}}\right\} \leq \bar{G}_{k}(t) \leq\left(g_{0}+1\right) \max \left\{t^{1+\delta}, t^{1+g_{0}}\right\} \quad \forall t \geq 0,
$$

by setting

$$
B_{\frac{5}{8}}^{-}:=B_{\frac{5}{8}} \cap\left\{\left|\nabla v_{k}-\nabla \xi_{k}\right|<1\right\} \text { and } B_{\frac{5}{8}}^{+}:=B_{\frac{5}{8}} \cap\left\{\left|\nabla v_{k}-\nabla w_{k}\right| \geq 1\right\},
$$

estimate (6.19) together with (6.20) give us

$$
C \cdot k^{-\frac{1+\delta}{2}} \geq\left(1+g_{0}\right)^{-2}\left(\int_{B_{\frac{5}{8}}^{-}}\left|\nabla v_{k}-\nabla \xi_{k}\right|^{g_{0}+1} \mathrm{~d} x+\int_{B_{\frac{5}{8}}^{+}}\left|\nabla v_{k}-\nabla w_{k}\right|^{\delta+1} \mathrm{~d} x\right) .
$$

Applying Hölder inequality in the above first integral we have

$$
\begin{aligned}
C \cdot k^{-\frac{1+\delta}{2}} \geq & \left(1+g_{0}\right)^{-2}\left|B_{\frac{5}{8}}\right|^{\frac{\delta-g_{0}}{1+\delta}}\left(\int_{B_{\frac{5}{8}}^{-}}\left|\nabla v_{k}-\nabla \xi_{k}\right|^{\delta+1} \mathrm{~d} x\right)^{\left(1+g_{0}\right) /(1+\delta)} \\
& +\left(1+g_{0}\right)^{-2} \int_{B_{\frac{5}{8}}^{+}}\left|\nabla v_{k}-\nabla \xi_{k}\right|^{\delta+1} \mathrm{~d} x
\end{aligned}
$$

Hence,

$$
\widetilde{C} \geq k^{\frac{1}{2\left(1+g_{0}\right)}} \int_{B_{\frac{5}{8}}}\left|\nabla v_{k}-\nabla \xi_{k}\right|^{1+\delta} \mathrm{d} x .
$$

By Poincaré inequality, we conclude that

$$
h_{k}:=v_{k}-\xi_{k} \longrightarrow 0 \text { strongly in } W_{0}^{1, \delta+1}\left(B_{\frac{5}{8}}\right) .
$$


Using one more time Theorem 5.1 and since interior Hölder estimates for $v_{k}$ is a consequence of Harnack inequality we conclude that there exists $\alpha \in(0,1)$ and constant $C^{\prime}=C^{\prime}\left(\delta, g_{0}, n, \rho_{0}, \rho, \varepsilon_{0}, \gamma, \Lambda_{0}, \mu_{0}, \mu, \alpha\right)>0$ such that

$$
\left\|v_{k}\right\|_{C^{\alpha}\left(B_{\frac{5}{9}}\right)},\left\|\xi_{k}\right\|_{C^{\alpha}\left(B_{\frac{5}{9}}\right)} \leq C^{\prime} .
$$

This imply that there exist functions $v_{\infty}, \xi_{\infty}$ such that

$$
\xi_{k} \rightarrow \xi_{\infty} \quad \text { uniformly in } B_{5 / 9} \quad \text { and } \quad v_{k} \rightarrow v_{\infty} \quad \text { uniformly in } B_{5 / 9} .
$$

So, from (6.21) we arrive at $v_{\infty}=\xi_{\infty}$. Once more by Harnack inequality

$$
\sup _{B_{1 / 2}} v_{k} \leq C_{0} \inf _{B_{1 / 2}} v_{k}
$$

where $C_{0}$ is a universal constant. Then, passing the limit in the second estimate of (6.15) and in (6.22), we have

$$
0<\frac{3 \widetilde{c}}{4 C_{0}} \leq \frac{1}{C_{0}} \sup _{B_{1 / 2}} v_{\infty} \leq \inf _{B_{1 / 2}} v_{\infty} \leq \xi_{\infty}(0)=0 .
$$

But this is a contradiction. Thus,

$$
u(x) \leq C_{1} \cdot \operatorname{dist}(x, \partial\{u>0\}), \quad \forall x \in \Omega^{\prime} .
$$

Suppose now that $0<d:=\operatorname{dist}\left(\Omega^{\prime}, \partial \Omega\right) \neq 1$. If $d>1$ we define

$$
\Omega^{*}:=\{x \in \Omega: \operatorname{dist}(x, \partial \Omega)>1\} .
$$

Notice that $\Omega^{*}$ is an open set that depends only on $\Omega$ and satisfies $\Omega^{\prime} \subseteq \Omega^{*}$ and $\operatorname{dist}\left(\Omega^{*}, \partial \Omega\right)=1$. Using the previous case for $\Omega^{*}$, there exists $C_{1}^{*}=C_{1}^{*}\left(n, \delta, g_{0}, q, \varepsilon_{0}\right.$, $\left.\gamma_{1}, \lambda_{1}, \mu_{1}, \rho_{1}, \Lambda_{1}^{M}, \Omega\right)>0$ such that

$$
u(x) \leq C_{1}^{*} \cdot \operatorname{dist}(x, \partial\{u>0\}) \leq \frac{C_{1}^{*} \cdot \operatorname{diam}(\Omega)}{\operatorname{dist}\left(\Omega^{\prime}, \partial \Omega\right)} \cdot \operatorname{dist}(x, \partial\{u>0\}), \quad \forall x \in \Omega^{\prime} .
$$

Hence, put $C_{1}=C_{1}^{*} \cdot \operatorname{diam}(\Omega)$. Finally assume that $d \in(0,1)$ and consider the following rescaling

$$
v(x):=u(\mathrm{~d} x), \quad x \in \Omega_{d}:=\left\{d^{-1} y: y \in \Omega\right\} .
$$

We have $\operatorname{dist}\left(\Omega_{d}^{\prime}, \partial \Omega_{d}\right)=1$. In addition, we observe that $v \in \mathcal{S}_{\varepsilon_{0}}\left(\Omega_{d}, M, \Lambda_{i}^{M}\right)$. In fact, if $u$ is a minimizer of

$$
\mathcal{J}_{G}(w, \Omega)=\int_{\Omega}\left[G(|\nabla w|)+f(x) H\left(w^{+}\right)+h(x) \chi_{\{w>0\}}\right] \mathrm{d} x,
$$

satisfying the conditions of Definition 2.3, then, $v$ is a minimizer of

$$
\mathcal{J}_{G_{d^{-1}}^{*}}\left(\xi, \Omega_{d}\right)=\int_{\Omega_{d}}\left[G_{d^{-1}}^{*}(|\nabla \xi|)+f(\mathrm{~d} x) H\left(\xi^{+}\right)+h(\mathrm{~d} x) \chi_{\{\xi>0\}}\right] \mathrm{d} x,
$$

where $G_{d^{-1}}^{*}(t)=G\left(d^{-1} t\right)$, and by Proposition 6.1 of [6], $G_{d^{-1}}^{*} \in \mathcal{G}\left(\delta, g_{0}, \varepsilon_{0}\right)$. Therefore,

$$
v(x) \leq C_{1} \cdot \operatorname{dist}(x, \partial\{v>0\}) .
$$


If $\partial\{u>0\}_{d}:=\left\{d^{-1} z: z \in \partial\{u>0\}\right\}$, we can translate this back in terms of $u$ and to conclude that

$$
u(y) \leq C_{1} \cdot \operatorname{dist}\left(d^{-1} y, \partial\{u>0\}_{d}\right)=\frac{C_{1}}{d} \cdot \operatorname{dist}(y, \partial\{u>0\}) .
$$

This proves (6.1). Estimate (6.2) follows essentially by (6.1) and local gradient estimates (3.3).

\section{Non-degeneracy of minimizers in the neighbourhood of the free boundary}

In this current section, we show that minimizers in a suitable class of $\mathcal{S}_{\varepsilon_{0}}\left(\Omega, M, \Lambda_{i}^{M}\right)$ grow at most linearly away from the free boundary. One more time, we assume that $q>n$. Now, we define a subclass of $\mathcal{S}_{\varepsilon_{0}}\left(\Omega, M, \Lambda_{i}^{M}\right)$.

Definition 7.1 Let $E_{0}>0$ and $\mu_{i} \geq \theta_{i}>0, i=1$, 2. A minimizer $u \in$ $\mathcal{S}_{\varepsilon_{0}}\left(\Omega, M, \Lambda_{i}^{M}, E_{0}, \theta_{i}\right)$ if

(i) $u \in \mathcal{S}_{\varepsilon_{0}}\left(\Omega, M, \Lambda_{i}^{M}\right)$, i.e. $u$ is a minimizer of a functional as (2.2) satisfying the conditions of Definition 2.3;

(ii) $0<\varepsilon_{0} \leq G(1) \leq E_{0}$ and;

(iii) $0<\theta_{i} \leq h_{i} \leq \mu_{i}, i=1,2$.

Theorem 7.1 (Nondegeneracy of minimizers) Let $u \in \mathcal{S}_{\varepsilon_{0}}\left(\Omega, M, \Lambda_{i}^{M}, E_{0}, \theta_{i}\right)$ and $\Omega^{\prime} \Subset \Omega$. Then, there exists a positive constant $c=c\left(n, \delta, g_{0}, q, \lambda_{1}, \Lambda_{1}^{M}, \theta_{1}, E_{0}, \Omega^{\prime}, \Omega\right)$ such that

$$
u\left(x_{0}\right) \geq c \cdot \operatorname{dist}\left(x_{0}, F^{+}(u)\right),
$$

for any point $x_{0} \in\{u>0\} \cap \Omega^{\prime}$. If $x_{0} \in\{u<0\} \cap \Omega^{\prime}$ then,

$$
u^{-}\left(x_{0}\right) \geq \widetilde{c} \cdot \operatorname{dist}\left(x_{0}, F^{-}(u)\right),
$$

for some $\tilde{c}=\tilde{c}\left(n, \delta, g_{0}, q, \lambda_{2}, \Lambda_{2}^{M}, \theta_{2}, E_{0}, \Omega^{\prime}, \Omega\right)>0$. Here $F^{-}(u):=\partial\{u<0\} \cap \Omega$.

Proof We prove (7.1). The proof of (7.2) follows the same guidelines. First, notice that it suffices to show such estimate for point $x_{0} \in\{u>0\} \cap \Omega^{\prime}$ that are close enough to the free boundary, i.e. satisfying

$$
0<\operatorname{dist}\left(x_{0}, F^{+}(u)\right)<\min \left\{d, \operatorname{dist}\left(\Omega^{\prime}, \partial \Omega\right)\right\}
$$

for some $d=d\left(n, \delta, g_{0}, q, \lambda_{1}, \Lambda_{1}^{M}, \theta_{1}, \varepsilon_{0}, E_{0}\right)>0$. Let $d_{0}:=\operatorname{dist}\left(x_{0}, \partial\{u>0\}\right)$. Clearly, we can assume that $0<d_{0} \leq 1$. We know that there exist functional $\mathcal{J}_{G}$ as in (2.2) such that $u$ is a minimizer of $\mathcal{J}_{G}$. Defining the rescaled function

$$
w(x):=\frac{u\left(x_{0}+d_{0} x\right)}{d_{0}}
$$

it is enough to show that $w(0) \geq c_{0}$. Now, we observe that $w$ is a local minimizer for the functional of the type

$$
\widetilde{\mathcal{J}}_{G}\left(v, B_{1}\right)=\int_{B_{1}}\left[G(|\nabla v|)+\tilde{f}_{1}(x) \tilde{H}_{i}\left(v^{+}\right)+\tilde{f}_{2}(x) \widetilde{H}_{i}\left(v^{-}\right)+Q\left(\tilde{h}_{1}, \tilde{h}_{2}\right)\right] \mathrm{d} x,
$$


where $\widetilde{f_{i}}(x)=f_{1}\left(x_{0}+d_{0} x\right), \widetilde{H}_{i}(t)=H_{i}\left(d_{0} \cdot t\right)$ and $\widetilde{h}_{i}(x)=h_{i}\left(x_{0}+d_{0} x\right)$. It follows by Corollary 5.1 that

$$
\mathcal{L}_{g} w=d_{0} \cdot f_{1}(x) H_{1}^{\prime}\left(d_{0} \cdot w\right) \text { in } B_{1} \subset\{w>0\} .
$$

By Lemma 2.2 of [18] we have that $g^{-1}(1) \leq 1 / \min \left\{\varepsilon_{0}^{1 / \delta}, \varepsilon_{0}^{1 / g_{0}}\right\}$ and using one more time Lemma 2.2 of [18] and Harnack inequality (Lemma 3.4) we arrive that, $\forall x \in B_{\frac{7}{8}}$,

$$
w(x) \leq C_{0}\left(w(0)+\Upsilon_{0}\right) \quad \text { where } \quad \Upsilon_{0}:=d_{0}^{\frac{q-n}{q g_{0}}} \frac{\left(\Lambda_{1}^{M} \cdot \lambda_{1}+1\right)^{1 / \delta}}{\min \left\{\varepsilon_{0}^{1 / \delta}, \varepsilon_{0}^{1 / g_{0}}\right\}} .
$$

Now, considering $\psi \in C^{\infty}\left(B_{1}(0)\right)$ such that

$$
\psi \equiv 1 \text { in } B_{1} \backslash B_{1 / 2}, \quad \psi \equiv 0 \text { in } B_{1 / 4}, \quad|\nabla \psi| \leq \widetilde{C} \quad \text { in } B_{1},
$$

we observe that

$$
\widetilde{\psi}:= \begin{cases}\min \left\{w, C_{0}\left(w(0)+\Upsilon_{0}\right) \psi\right\} & \text { in } B_{\frac{7}{8}} \\ w & \text { in } B_{1} \backslash B_{\frac{7}{8}}\end{cases}
$$

belongs to $K_{w}=\left\{v \in W^{1, G}\left(B_{1}\right): v=w\right.$ on $\left.\partial B_{1}\right\}$ and $0 \leq \widetilde{\psi} \leq w$. By minimality of $w$, definition of $\psi$, mean value theorem, Hölder and Young inequalities we have

$$
\begin{aligned}
& \int_{\{\tilde{\psi}<w\}}(G(|\nabla \widetilde{\psi}|)-G(|\nabla w|) \mathrm{d} x \\
& \geq-\Lambda_{1}^{M} d_{0} \int_{\{\tilde{\psi}<w\}}\left|\tilde{f}_{1}\right||w-\widetilde{\psi}| \mathrm{d} x+\theta_{1} \int_{\{\tilde{\psi}<w\}}\left(\chi_{\{w>0\}}-\chi_{\{\tilde{\psi}>0\}}\right) \mathrm{d} x \\
& \geq-C_{1} \cdot d_{0}^{1-\frac{n}{q}}\left(\int_{\{\widetilde{\psi}<w\}}|w-\widetilde{\psi}|^{1+\delta} \mathrm{d} x\right)^{\frac{1}{1+\delta}}+\theta_{1}\left|B_{1 / 4}\right| . \\
& \geq-C_{1} \cdot d_{0}^{1-\frac{n}{q}}\left[C_{0}^{1+\delta}\left(w(0)+\Upsilon_{0}\right)^{1+\delta}+\frac{\delta}{1+\delta}\right]+\theta_{1}\left|B_{1 / 4}\right|,
\end{aligned}
$$

where $C_{1}=\Lambda_{1}^{M} \lambda_{1}\left|B_{1}\right|^{\frac{\delta}{1+\delta}-\frac{1}{q}}$. On the other hand,

$\int_{\{\tilde{\psi}<w\}}\left(G(|\nabla \tilde{\psi}|)-G(|\nabla w|) \mathrm{d} x \leq \int_{B_{1}} G\left(C_{0}\left(w(0)+\Upsilon_{0}\right)|\nabla \psi|\right) \mathrm{d} x \leq C_{2} \cdot\left(w(0)^{\tau}+\Upsilon_{0}^{\tau}\right)\right.$,

where $C_{2}=\left(1+g_{0}\right)\left[2 C_{0}(\widetilde{C}+1)\right]{ }^{1+g_{0}} E_{0}\left|B_{1}\right|$ and $\tau=1+\delta$ or $\tau=1+g_{0}$. If $\tau=1+\delta$ follows by above estimates that

$$
C_{4} w^{1+\delta}(0) \leq \theta_{1}\left|B_{1 / 4}\right|-\frac{C_{1} \delta}{1+\delta} d_{0}^{1-\frac{n}{q}}-C_{4} \Upsilon_{0}^{1+\delta},
$$

for $C_{4}=2^{1+g_{0}} C_{0}^{1+\delta} C_{1}+C_{2}$. If $\tau=1+g_{0}$, then using again Young inequality,

$$
C_{4} w^{1+g_{0}}(0) \leq \theta_{1}\left|B_{1 / 4}\right|-C_{1}\left(\frac{C_{0}^{1+\delta}\left(g_{0}-\delta\right)}{1+g_{0}}+\frac{\delta}{1+\delta}\right) d_{0}^{1-\frac{n}{q}}-C_{4} \Upsilon_{0}^{1+g_{0}} .
$$

Now, by above estimates, we choose $d_{0}$ such that

$$
\frac{\theta_{1}}{8}\left|B_{1 / 4}\right| \leq \max \left\{C_{1}\left(\frac{C_{0}^{1+\delta}\left(g_{0}-\delta\right)}{1+g_{0}}+\frac{\delta}{1+\delta}\right) d_{0}^{1-\frac{n}{q}}, C_{4} \Upsilon_{0}^{1+\delta}\right\} \leq \frac{\theta_{1}}{4}\left|B_{1 / 4}\right|
$$


Thus, by (7.3) and (7.4)

$$
w(0) \geq \max \left\{\left(\frac{\theta_{1}}{2 C_{4}}\left|B_{1 / 4}\right|\right)^{\frac{1}{1+\delta}},\left(\frac{\theta_{1}}{2 C_{4}}\left|B_{1 / 4}\right|\right)^{\frac{1}{1+g_{0}}}\right\} .
$$

In the presence of Lipschitz regularity, we obtain the strong non-degeneracy for minimizers. Before we provide the following key step.

Lemma 7.1 Let $u$ be a locally Lipschitz function in $\mathcal{S}_{\varepsilon_{0}}\left(\Omega, M, \Lambda_{i}^{M}, E_{0}, \theta_{i}\right)$. Then, for any $\Omega^{\prime} \subset \subset \Omega$, there exist constants $r_{0}, \delta_{0}>0$ and $\gamma_{0} \in(0,1]$ such that if $x_{0} \in\{u>0\} \cap \Omega^{\prime}$ and $\operatorname{dist}\left(x_{0}, F^{+}(u)\right)=r \leq r_{0}$, the following estimate is satisfied

$$
u\left(x_{1}\right) \geq\left(1+\delta_{0}\right) u\left(x_{0}\right)
$$

for some $x_{1} \in \bar{B}_{r}\left(x_{0}\right) \backslash B_{\gamma_{0} r}\left(x_{0}\right)$.

Remark 7.1 We point out that if $f_{1} \equiv 0$ we can take $\gamma_{0}=1$ and hence $x_{1} \in \partial B_{r}\left(x_{0}\right)$. The constants $r_{0}, \delta_{0}$ and $\gamma_{0}$ depend on $n, \delta, g_{0}, q, \lambda_{1}, \Lambda_{1}^{M}, \theta_{1}, E_{0}, \Omega^{\prime}, \Omega$ and $\|\nabla u\|_{L^{\infty}\left(\Omega^{\prime \prime}\right)}$ where

$$
\Omega^{\prime \prime}:=\left\{x \in \Omega: \operatorname{dist}(x, \partial \Omega)>\frac{1}{2} \operatorname{dist}\left(\Omega^{\prime}, \partial \Omega\right)\right\} .
$$

Similar estimate as in (7.5) holds if $x_{0} \in\{u<0\} \cap \Omega^{\prime}$. Precisely,

$$
u^{-}\left(x_{1}\right) \geq\left(1+\widetilde{\delta}_{0}\right) u^{-}\left(x_{0}\right),
$$

where $\operatorname{dist}\left(x_{0}, F^{-}(u)\right)=r \leq \widetilde{r}_{0}$ and $x_{1} \in \bar{B}_{r}\left(x_{0}\right) \backslash B_{\tilde{\gamma}_{0} r}\left(x_{0}\right)$ for some constants $\widetilde{\delta}_{0}, \widetilde{r}_{0}>0$ and $\tilde{\gamma}_{0} \in(0,1]$ depend on $n, \delta, g_{0}, q, \lambda_{2}, \Lambda_{2}^{M}, \theta_{2}, E_{0}, \Omega^{\prime}, \Omega$ and $\|\nabla u\|_{L^{\infty}\left(\Omega^{\prime \prime}\right)}$.

Proof of Lemma 7.1 Taking into account the linear growth for $u \in \mathcal{S}_{\varepsilon_{0}}\left(\Omega, M, \Lambda_{i}^{M}, E_{0}, \theta_{i}\right)$, this result can be proven as a variant of Proposition 4.3 in [19] since the crucial argument is based on an iteration performed via weak Harnack inequality also available for our operator $\mathcal{L}_{g}$ (see Theorem 1.3 and Section 4 in [17]).

Theorem 7.2 (Strong non-degeneracy estimate) Let $u$ be a locally Lipschitz function in $\mathcal{S}_{\varepsilon_{0}}\left(\Omega, M, \Lambda_{i}^{M}, E_{0}, \theta_{i}\right)$. Then, for any $\Omega^{\prime} \subset \subset \Omega$ there exists a positive constant $c_{0}$ such that, for any $0<r<\operatorname{dist}\left(\Omega^{\prime}, \partial \Omega\right)$,

$$
\sup _{B_{r}\left(x_{0}\right)} u^{+} \geq c_{0} \cdot r
$$

provided $x_{0} \in \overline{\{u>0\}} \cap \Omega^{\prime}$. If we have $x_{0} \in \overline{\{u<0\}} \cap \Omega^{\prime}$, then there exists $\widetilde{c}_{0}>0$ such that

$$
\sup _{B_{r}\left(x_{0}\right)} u^{-} \geq \widetilde{c}_{0} \cdot r
$$

The constants $c_{0}$ and $\widetilde{c}_{0}$ depend on $n, \delta, g_{0}, q, \lambda_{i}, \Lambda_{i}^{M}, \theta_{i}, E_{0}, \Omega^{\prime}, \Omega$ and $\|\nabla u\|_{L^{\infty}\left(\Omega^{\prime \prime}\right)}$.

Proof The proof follows the same lines of Theorem 4.1 of [19]. 


\section{Log-Lipschitz regularity in the class $\mathcal{S}_{\varepsilon_{0}}\left(\Omega, M, \Lambda_{i}^{M}\right)$ - non-singular operators}

It is a well-known fact among specialists of this research area that the Lipschitz continuity of minimizers plays a important role on the development of the study of the free boundary. But from previous considerations made in Sect. 1, such regularity may be unknown in more general scenarios. However, in [15], the authors were able to show asymptotically optimal result, i.e. local log-Lipschitz regularity. Motivated by this fact, we investigate suitable conditions to the asymptotic optimal regularity for local minimizers in the class $\mathcal{S}_{\varepsilon_{0}}\left(\Omega, M, \Lambda_{i}^{M}\right)$. We observe that in [20] (Orlicz-Sobolev context), this result has been proven for local minima of (1.3) since $\frac{g(t)}{t}$ is nondecreasing. We notice that such restriction hide precisely that the $\mathcal{L}_{g}$-operator is, in certain sense, non-singular. This is justified by the following definition and next lemma.

Definition 8.1 Let $G \in \mathcal{G}\left(\delta, g_{0}\right)$ and $G^{\prime}=g$. We say that the $\mathcal{L}_{g}$-operator is:

(i) (strongly) singular operator when $g_{0}<1$;

(ii) weakly singular operator if $0<\delta<g_{0}=1$;

(iii) non-singular operator when $\delta \geq 1$;

By simplicity, we call (strongly) singular operators only singular operators. We observe still that if $\delta>1$ then $\mathcal{L}_{g}$ is degenerate. The above lemma provides a criterion for new concepts. In the cases where $0<\delta<1<g_{0}$ the $\mathcal{L}_{g}$-operator does not enjoy a character purely singular or degenerate.

Lemma 8.1 Let $G \in \mathcal{G}\left(\delta, g_{0}\right)$ and $G^{\prime}=g$. Then,

(i) $\mathcal{L}_{g}$ is singular if $\frac{g(t)}{t}$ is decreasing for $t>0$.

(ii) $\mathcal{L}_{g}$ is weakly singular if and only if $\mathcal{L}_{g}$ is not singular, $\frac{g(t)}{t}$ is nonincreasing for $t>0$ and $G$ is not a power of $t$.

(iii) $\mathcal{L}_{g}$ is non-singular if and only if $\frac{g(t)}{t}$ is nondecreasing for $t>0$.

Proof (i) In fact,

$$
\mathcal{L}_{g} \text { is singular } \Longrightarrow \frac{t g^{\prime}(t)}{g(t)}<1 \Longrightarrow\left(\frac{g(t)}{t}\right)^{\prime}<0 \Longrightarrow \frac{g(t)}{t} \text { is decreasing for } t>0 .
$$

(ii) Suppose $\mathcal{L}_{g}$ is weakly singular. By definition $\mathcal{L}_{g}$ is not singular. Still, $\left(\frac{g(t)}{t}\right)^{\prime} \leq 0$ and hence $\frac{g(t)}{t}$ is nonincreasing for $t>0$. Moreover, since $\delta<1, \frac{t^{\prime}(t)}{g(t)}$ can not be a constant. This implies that $G$ is not a power of $t$. Conversely, assume that $\mathcal{L}_{g}$ is not singular, $\frac{g(t)}{t}$ is nonincreasing for $t>0$ and $G$ is not a power of $t$. This implies that $g_{0} \geq 1, \frac{t g^{\prime}(t)}{g(t)} \leq 1$ and $0<\delta<g_{0}$. This shows that $\mathcal{L}_{g}$ is a weakly singular operator.

(iii) Indeed, for $t>0$ we have that $\left(\frac{g(t)}{t}\right)^{\prime} \geq 0$ if and only if $\delta \geq 1$.

The next lemma is simple, but an important fact for non-singular $\mathcal{L}_{g}$ operators.

Lemma 8.2 Let $u$ a minimizer of a functional of the type $\mathcal{J}_{G}$ with $G \in \mathcal{G}\left(\delta, g_{0}\right)$ where $\delta \geq 1$. Assume $B_{r}\left(x_{0}\right) \subset \Omega$ and $w$ be the solution to

$$
\mathcal{L}_{g} w=0 \text { in } B_{r}\left(x_{0}\right) \text { and } w=u \text { on } \partial B_{r}\left(x_{0}\right) .
$$


Then,

$$
\int_{B_{r}\left(x_{0}\right)}(G(|\nabla u|)-G(|\nabla w|)) \mathrm{d} x \geq C\left(\delta, g_{0}\right) \int_{B_{r}\left(x_{0}\right)} G(|\nabla u-\nabla w|) \mathrm{d} x .
$$

Proof By notation of Theorem 5.1 we have

$$
\begin{aligned}
& \int_{A_{1}} F(|\nabla u|)|\nabla u-\nabla v|^{2} \mathrm{~d} x \geq 2^{1-g_{0}} \int_{A_{1}} F(|\nabla u-\nabla v|)|\nabla u-\nabla v|^{2} \mathrm{~d} x \\
& \quad \geq 2^{1-g_{0}} \int_{A_{1}} G(|\nabla u-\nabla v|) \mathrm{d} x .
\end{aligned}
$$

Now, the proof follows by combination of the above estimate and (5.1) in Theorem 5.1.

Remark 8.1 We note that if $\delta<1$, then

$$
\int_{A_{1}} F(|\nabla u|)|\nabla u-\nabla v|^{2} \mathrm{~d} x \leq 2^{1-g_{0}} \int_{A_{1}} G(|\nabla u-\nabla v|) \mathrm{d} x .
$$

The next lemma was proved in [20] (see Lemma 4.1).

Lemma 8.3 Let $u, w \in W^{1, G}\left(B_{R}\right)$ where $\mathcal{L}_{g} w=0$ in $B_{R}$ in the distributional sense. Then, there exist constants $C>0$ and $\sigma \in(0,1)$ depending only on $n, \delta, g_{0}$ such that, for all $0<r \leq R$ holds

$\int_{B_{r}} G\left(\left|\nabla u-(\nabla u)_{r}\right|\right) \mathrm{d} x \leq C\left[\left(\frac{r}{R}\right)^{n+\sigma} \int_{B_{R}} G\left(\left|\nabla u-(\nabla u)_{R}\right|\right) \mathrm{d} x+\int_{B_{R}} G(|\nabla u-\nabla w|) \mathrm{d} x\right]$,

where, for any function $\psi \in L^{1}\left(B_{R}\right)$ and $0<s \leq R$,

$$
(\psi)_{s}:=f_{B_{s}}|\psi(x)| \mathrm{d} x .
$$

We now have all the ingredients to show that the gradient of local minimizers are locally $\mathrm{BMO}$ for non-singular $\mathcal{L}_{g}$ operators. In the sequel, we assume that $q \geq n$ and the assumptions in the Remark 4.1.

Proof of Theorem 2.1 Let $y \in \Omega^{\prime}$ and $0<r<R=\operatorname{dist}\left(\Omega^{\prime}, \partial \Omega\right)$. By simplicity assume that $y=0$. Consider $w: B_{R} \rightarrow \mathbb{R}$ the solution to

$$
\mathcal{L}_{g} w=0 \text { in } B_{R} \text { and } u=w \text { on } \partial B_{R} .
$$

By Lemma 8.2 and using the minimality of $u$ we have that

$$
\begin{aligned}
& \int_{B_{r}} G(|\nabla u-\nabla w|) \mathrm{d} x \leq \frac{1}{C_{1}}\left[C_{2} R^{n}+\int_{B_{R}} f_{1}\left(H_{1}\left(u^{+}\right)-H_{1}\left(w^{+}\right)\right) \mathrm{d} x\right. \\
& \left.\quad+\int_{B_{R}} f_{2}\left(H_{2}\left(u^{-}\right)-H_{2}\left(w^{-}\right)\right) \mathrm{d} x\right],
\end{aligned}
$$

where $C_{1}=C_{1}\left(\delta, g_{0}\right)$ and $C_{2}=C_{2}\left(n, \mu_{1}, \mu_{2}\right)$. By maximum principle we know that $\sup _{B_{R}}|w| \leq M$. Thus, by mean value theorem

$$
\begin{aligned}
& \int_{B_{R}} f_{1}\left(H_{1}\left(u^{+}\right)-H_{1}\left(w^{+}\right)\right) \mathrm{d} x+\int_{B_{R}} f_{2}\left(H_{2}\left(u^{-}\right)-H_{2}\left(w^{-}\right)\right) \mathrm{d} x \\
& \quad \leq\left(\Lambda_{1}^{M}+\Lambda_{2}^{M}\right) \cdot \int_{B_{R}}\left(\left|f_{1}\right|+\left|f_{2}\right|\right)|u-w| \mathrm{d} x .
\end{aligned}
$$


Now, by Hölder, Poincaré and Young inequalities we arrive at

$$
\begin{aligned}
\int_{B_{R}}\left(\left|f_{1}\right|+\left|f_{2}\right|\right)|u-w| \mathrm{d} x & \leq\left(|| f_{1}\left\|_{L^{q}\left(B_{R}\right)}+\right\| f_{2} \|_{L^{q}\left(B_{R}\right)}\right)\left|B_{R}\right|^{\frac{\delta}{1+\delta}-\frac{1}{q}}\|u-w\|_{L^{1+\delta}\left(B_{R}\right)} \\
& \leq C(n)\left(\lambda_{1}+\lambda_{2}\right)|\Omega|^{\frac{q-n}{q n}}\left|R^{\frac{n \delta}{1+\delta}}\right| \mid \nabla u-\nabla w \|_{L^{1+\delta}\left(B_{R}\right)} \\
& \leq \frac{C_{4}}{c^{\frac{1+\delta}{\delta}}} \cdot R^{n}+c^{1+\delta} \int_{B_{R}}|\nabla u-\nabla w|^{1+\delta} \mathrm{d} x,
\end{aligned}
$$

where $c>0$ will be chosen a posteriori. Now, by $(G-2)$

$$
\begin{aligned}
\int_{B_{R}}|\nabla u-\nabla w|^{1+\delta} \mathrm{d} x & \leq \int_{\{|\nabla u-\nabla w|<1\}}|\nabla u-\nabla w|^{1+\delta} \mathrm{d} x+\int_{\{|\nabla u-\nabla w| \geq 1\}}|\nabla u-\nabla w|^{1+\delta} \mathrm{d} x \\
& \leq C(n) R^{n}+\frac{\left(1+g_{0}\right)}{\varepsilon_{0}} \int_{B_{R}} G(|\nabla u-\nabla w|) \mathrm{d} x .
\end{aligned}
$$

Combining the above estimates, we obtain

$$
\int_{B_{r}} G(|\nabla u-\nabla w|) \mathrm{d} x \leq C_{5} \cdot \varepsilon_{0}^{-1 / \delta} \cdot R^{n}+\frac{1}{2} \int_{B_{r}} G(|\nabla u-\nabla w|) \mathrm{d} x,
$$

where $c=\left[\frac{C_{1} \varepsilon_{0}}{2\left(1+g_{0}\right) C_{3}}\right]^{\frac{1}{1+\delta}}$. Now, by Lemma 8.3, we have

$$
\int_{B_{r}} G\left(\left|\nabla u-(\nabla u)_{r}\right|\right) \mathrm{d} x \leq C\left[\left(\frac{r}{R}\right)^{n+\sigma} \int_{B_{r}} G\left(\left|\nabla u-(\nabla u)_{R}\right|\right) \mathrm{d} x+2 C_{5} \cdot \varepsilon_{0}^{-1 / \delta} \cdot R^{n}\right] .
$$

Using Lemma 2.7 of [15] and Remark 4.3 follows that

$$
\begin{aligned}
\int_{B_{r}} G\left(\left|\nabla u-(\nabla u)_{r}\right|\right) \mathrm{d} x & \leq C_{6}\left(R^{-n} \int_{B_{R}} G\left(\left|\nabla u-(\nabla u)_{R}\right|\right) \mathrm{d} x+\varepsilon_{0}^{-1 / \delta} \cdot R^{n}\right) r^{n} \\
& \leq C_{7}\left(R^{-n}+\operatorname{diam}(\Omega)^{n} \cdot \varepsilon_{0}^{-1 / \delta}\right) r^{n},
\end{aligned}
$$

where $C_{7}=C_{7}\left(n, \delta, g_{0}, q, M, \lambda_{i}, \mu_{i}, \Lambda_{i, M},|\Omega|\right) \geq 1$. Finally, by Jensen inequality and (G - 2) we conclude that

$$
f_{B_{r}}\left|\nabla u-(\nabla u)_{r}\right| \mathrm{d} x \leq \frac{C_{8}}{\varepsilon_{0}} \cdot\left(\operatorname{dist}\left(\Omega^{\prime}, \partial \Omega\right)^{-n}+\operatorname{diam}(\Omega)^{n} \cdot \varepsilon_{0}^{-1 / \delta}\right)^{\frac{1}{1+\delta}},
$$

where $C_{8}=\left(1+g_{0}\right) C_{7}^{\frac{1}{1+\delta}}$. This proves (2.4). The estimate (2.5) follows immediately by Lemma 1 of [5].

\section{Compactness result and Invariance of Dini type control under suitable Scalings}

In the proof of Theorem 2.2 (main result), the use of a compactness approach is fundamental. We point out that in our context for classes, we need two compactness results, more precisely, compactness for minimizers and $N$-functions. The Hölder regularity for minimizers is sufficient for our purposes. In the case of $N$-functions, we need that the limit of $\mathrm{N}$-functions considered here continue satisfying the Lieberman conditions (PC) and (QC). 
Unfortunately, the classes $\mathcal{G}\left(\delta, g_{0}\right)$ and $\mathcal{G}\left(\delta, g_{0}, \varepsilon_{0}\right)$ are weak to provide the compactness desired. For instance, consider the following family $\left\{G_{N}\right\}$ of $N$-functions:

$$
G_{N}(t)= \begin{cases}\frac{3}{4} t^{2} & \text { if } 0 \leq t \leq 2 \\ \frac{(4+5 N)}{12} t^{3}-\frac{(5+10 N)}{4} t^{2}+(4+5 n) t-\frac{(8+10 N)}{3} & \text { if } 2 \leq t \leq 2+\frac{1}{N} \\ \frac{1}{3} t^{3}-\frac{(5+4 N)}{4 N} t+\frac{7}{3}+\frac{5}{2 N}+\frac{5}{12 N^{2}} & \text { if } 2+\frac{1}{N} \leq t .\end{cases}
$$

We see that $G_{N} \in \mathcal{G}(1,7,3 / 4)$ for all $N \in \mathbb{N}$ and $G_{N} \rightarrow G_{\infty}$ uniformly in the compact sets of $[0,+\infty)$ where

$$
G_{\infty}(t)= \begin{cases}\frac{3}{4} t^{2} & \text { if } 0 \leq t \leq 2 \\ \frac{1}{3} t^{3}-t+\frac{7}{3} & \text { for } t \geq 2\end{cases}
$$

Since $G_{\infty} \in C^{1,1}([0, \infty)) \backslash C^{2}[0, \infty)$ follows that $G_{\infty} \notin \mathcal{G}\left(\delta, g_{0}\right)$ for whatever $0<\delta \leq g_{0}$.

In view of this, we need a suitable uniform modulus of continuity for $Q_{g}$. In this section, we show that the Dini type control meets our interests before we prove a lemma about Dini continuity and uniform convergence.

Lemma 9.1 Let $\left\{f_{N}\right\}$ a locally bounded sequence of continuous functions defined in the same domain. Assume that there exist modulus of continuity $\left\{\omega_{N}\right\}$ such that, for some positive constant $D_{1}$,

$$
\left|f_{N}(x)-f_{N}(y)\right| \leq D_{1} \cdot \omega_{N}(|x-y|), \quad \forall N \in \mathbb{N}
$$

and, for some $L, D_{2}>0$,

$$
\int_{0}^{L} \frac{\omega_{N}(t)}{t} \mathrm{~d} t \leq D_{2}, \quad \forall N \in \mathbb{N} .
$$

Then, up to subsequence, $\left\{f_{N}\right\}$ converges locally uniformly for a function $f$.

Proof By Arzelá-Ascoli theorem, it is sufficient to prove that $\left\{\omega_{N}\right\}$ is equicontinuous. Suppose that this does not occurs. Then, there exists a subsequence $\left\{\omega_{N_{j}}\right\}$ and $t_{N_{j}} \rightarrow 0$ such that, for some $\varepsilon>0, \omega_{N_{j}}\left(t_{N_{j}}\right) \geq \varepsilon, \forall j \in \mathbb{N}$. Clearly we can assume that $0<t_{N_{j}}<L$. In particular,

$$
D_{2} \geq \int_{t_{N_{j}}}^{L} \frac{\omega_{N_{j}}(t)}{t} \mathrm{~d} t \geq \varepsilon \int_{t_{N_{j}}}^{L} \frac{1}{t} \mathrm{~d} t=\varepsilon\left(\ln (L)-\ln \left(t_{N_{j}}\right)\right) .
$$

This is a contradiction for $t_{N_{j}}<<1$.

Theorem 9.1 (Compactness for $N$-functions) Let

$$
\vartheta\left(\varepsilon_{0}, E_{0}\right):=\left\{G \in \mathcal{G}_{\xi_{i} \cdot D}\left(\delta, g_{0}, \varepsilon_{0}\right): G(1) \leq E_{0}\right\} .
$$

If $\left\{G_{N}\right\} \subset \vartheta\left(\varepsilon_{0}, E_{0}\right)$ then, up to subsequence, $\left\{G_{N}\right\}$ converges to a $N$-function $G_{\infty} \in$ $\mathcal{G}\left(\delta, g_{0}\right)$ in $C^{2}$ topology on compact subsets of $(0,+\infty)$ and in the $C^{1}$ topology on compact subsets of $[0,+\infty)$. 
Proof Let $\left\{G_{N}\right\}$ be a sequence in $\vartheta\left(\varepsilon_{0}, E_{0}\right)$. By properties of $G_{N}$,

$$
\begin{aligned}
& \frac{\varepsilon_{0}}{\left(g_{0}+1\right)} \min \left\{t^{1+\delta}, t^{1+g_{0}}\right\} \leq G_{N}(t) \leq E_{0}\left(g_{0}+1\right) \max \left\{t^{1+\delta}, t^{1+g_{0}}\right\} \quad \forall t \geq 0, \forall N>0, \\
& \varepsilon_{0} \min \left\{t^{\delta}, t^{g_{0}}\right\} \leq g_{N}(t) \leq\left(1+g_{0}\right) E_{0} \max \left\{t^{\delta}, t^{g_{0}}\right\} \quad \forall t \geq 0, \forall N>0, \\
& 0<g_{N}^{\prime}(t) \leq g_{0}\left(1+g_{0}\right) E_{0} \max \left\{t^{\delta-1}, t^{g_{0}-1}\right\} \quad \forall t>0, \forall N>0,
\end{aligned}
$$

where $g_{N}=G_{N}^{\prime}$. Once $G_{N}$ satisfies (DTC) follows that $g_{N}^{\prime}$ is locally Dini continuous with uniform Dini constant. Indeed, let $K \Subset(0,+\infty)$ a closed interval. We have that, $g_{N}^{\prime}(t)=Q_{g_{N}}(t) \cdot \frac{g_{N}(t)}{t}$. By the way, $\left\{g_{N}^{\prime}\right\}$ is uniform bounded in $K$ and if $\omega_{N}, \widetilde{\omega}_{N}$ are the modulus of continuity of $g_{N}^{\prime}$ and $\frac{g_{N}(t)}{t}$ respectively, then by (9.2),

$$
\omega_{N}(t) \leq C_{1} \cdot \omega_{g_{N}}^{l, L}(t)+g_{0} \cdot \widetilde{\omega}_{N}(t),
$$

where $K=[l, L]$ and $C_{1}=C_{1}\left(\delta, g_{0}, E_{0}, l, L\right)>0$. We know that $\frac{g_{N}(t)}{t}$ is locally Lipschitz and holds

$$
\widetilde{\omega}_{N}(t) \leq \frac{\left(1+g_{0}\right) C_{1}}{l} \cdot t, \quad 0 \leq t \leq L-l .
$$

Thus, combination of (9.4), (9.5) and (DTC) we assure that

$$
\int_{0}^{L-l} \frac{\omega_{N}(t)}{t} \mathrm{~d} t \leq C_{1} \cdot C_{1}^{*} \cdot \xi_{1}\left(\frac{L}{l}\right) \cdot \xi_{2}\left(\frac{L-l}{l}\right)+\frac{\left(1+g_{0}\right) C_{1}}{l}(L-l), \quad \forall N \in \mathbb{N} .
$$

Since $G_{N}$ is uniform bounded in $C^{2}(K)$ by $(9.1),(9.2)$ and (9.3) and holds the above estimate, follows by Lemma 9.1 the existence of a $C^{2}$-function $\bar{G}_{\infty}:(0, \infty) \rightarrow \mathbb{R}$ such that, up to subsequence, $G_{N} \rightarrow G_{\infty}$ in $C^{2}$ topology for any compact interval of $(0, \infty)$. Note that we can to extend $G_{\infty}$ to become $C^{1}[0, \infty)$. In fact, passing to the limit in (9.1), for $0<t<1$, we have $G_{\infty}(t) \leq L_{0}\left(1+g_{0}\right) \cdot t^{1+\delta}$, which yields $\lim _{t \rightarrow 0^{+}} G_{\infty}(t)=0$. By

$0 \leq \lim _{t \rightarrow 0^{+}} \frac{G_{\infty}(t)}{t} \leq E_{0}\left(1+g_{0}\right) \lim _{t \rightarrow 0^{+}} t^{\delta}=0 \quad$ and $\quad 0<t<1 \Longrightarrow g_{\infty}(t) \leq\left(1+g_{0}\right) E_{0} t^{\delta}$, we see that $G_{\infty} \in C^{1}[0, \infty)$. Thus, follows that $G_{\infty} \in \mathcal{G}\left(\delta, g_{0}\right)$ and $G_{N} \rightarrow G_{\infty}$ in $C_{l o c}^{1}[0, \infty)$.

Remark 9.1 By [6] we know that $\mathcal{G}_{\text {Lip }}\left(\delta, g_{0}\right) \varsubsetneqq \mathcal{G}_{\beta \cdot H}\left(\delta, g_{0}\right) \varsubsetneqq \mathcal{G}_{\epsilon \cdot H}\left(\delta, g_{0}\right)$ for any $0<$ $\epsilon<\beta<1$. We can check that there exists a class $\mathcal{G}_{\left(\xi_{1}, \xi_{2}\right) \cdot D}\left(\delta, g_{0}\right)$ such that $\mathcal{G}_{\beta \cdot H}\left(\delta, g_{0}\right) \varsubsetneqq$ $\mathcal{G}_{\left(\xi_{1}, \xi_{2}\right) \cdot D}\left(\delta, g_{0}\right)$ for any $\beta \in(0,1]$. In fact, let $G \in \mathcal{G}_{\beta \cdot H}\left(\delta, g_{0}\right)$. Then, $Q_{g}$ is locally Hölder continuous in $(0, \infty)$ and for some constant $C_{\beta}=C_{\beta}\left(\delta, g_{0}, \beta\right)>0$ holds $\left[Q_{g}\right]_{C^{0, \beta}([l, L])} \leq$ $\frac{C_{\beta}}{l^{\beta}}$. In particular, $\omega_{g}^{l, L}(t) \leq \frac{C_{\beta}}{l^{\beta}} t^{\beta}$ and hence

$$
\int_{0}^{L-l} \frac{\omega_{g}^{l, L}(t)}{t} \mathrm{~d} t \leq \frac{C_{\beta}}{\beta} \cdot\left(\frac{L-l}{l}\right)^{\beta} .
$$

Since $\beta \in(0,1]$ is fixed, we prove that $G \in \mathcal{G}_{\left(\xi_{1}, \xi_{2}\right) \cdot D}\left(\delta, g_{0}\right)$ for

$$
C_{1}^{*}=\frac{C_{\beta}}{\beta}, \quad \xi_{1} \equiv 1, \quad \xi_{2}(t)=t^{\beta} .
$$

Finally, we observe that

$$
\mathcal{G}_{\beta \cdot H}\left(\delta, g_{0}\right) \varsubsetneqq \bigcup_{\gamma \in(0,1]} \mathcal{G}_{\gamma \cdot H}\left(\delta, g_{0}\right) \subseteq \mathcal{G}_{\left(\xi_{1}, \xi_{2}\right) \cdot D}\left(\delta, g_{0}\right) .
$$


Example 9.1 In this example, we show that there exist a considerable number of $N$-functions in $\mathcal{G}\left(\delta, g_{0}, \varepsilon_{0}\right)$ which do not belong to any $\mathcal{G}_{\gamma \cdot H}\left(\delta, g_{0}, \varepsilon_{0}\right)$ but belongs to $\mathcal{G}_{\left(\xi_{1}, \xi_{2}\right) \cdot D}\left(\delta, g_{0}, \varepsilon_{0}\right)$. In particular, we show that, for suitable $0<\delta \leq g_{0}$, and functions $\xi_{1}, \xi_{2}$,

$$
\bigcup_{\gamma \in(0,1]} \mathcal{G}_{\gamma \cdot H}\left(\delta, g_{0}\right) \varsubsetneqq \mathcal{G}_{\left(\xi_{1}, \xi_{2}\right) \cdot D}\left(\delta, g_{0}\right) .
$$

Indeed, let $v \in \mathbb{N}$ fixed arbitrarily and $g_{v}:[0, \infty) \rightarrow[0, \infty)$ the following function

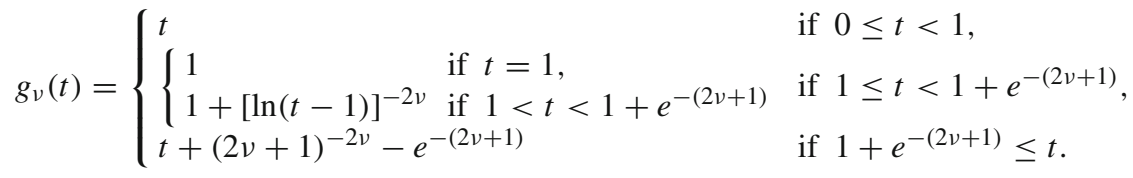

Clearly $g_{v}$ is continuous and increasing in $[0, \infty)$. Now, define for all $t \geq 0$,

$$
g(t)=\int_{0}^{t} g_{v}(s) \mathrm{d} s \quad \text { and } \quad G(t)=\int_{0}^{t} g(s) \mathrm{d} s .
$$

Simple but long computations show that $G \in \mathcal{G}\left(1, g_{0}, 1\right)$, where $g_{0}=g_{0}(v)>1$. Moreover, for any $\beta \in(0,1]$, we have that

$$
\lim _{t \rightarrow 1^{+}} \frac{\left|g_{v}(t)-g_{v}(0)\right|}{t^{\beta}}=\infty .
$$

This proves that $G \notin \mathcal{G}_{\gamma \cdot H}\left(\delta, g_{0}, \varepsilon_{0}\right)$ whatever $\beta \in(0,1)$. On the other hand, there exist functions $\xi_{1}, \xi_{2}$ such that $G \in \mathcal{G}_{\left(\xi_{1}, \xi_{2}\right) \cdot D}\left(1, g_{0}, 1\right)$. In fact, first we note that, if $0<l<L \leq 1$, then $\omega_{g}^{l, L} \equiv 0$ satisfying (DTC) in this interval. Still, if $h(t)=\frac{t}{g(t)}$ and

$$
\begin{aligned}
& \omega_{v}^{l, L}(t):=\sup \left\{\left|g_{v}(x)-g_{v}(y)\right|: l \leq x, y \leq L \text { and }|x-y| \leq t\right\}, \\
& \omega_{h}^{l, L}(t):=\sup \{|h(x)-h(y)|: l \leq x, y \leq L \text { and }|x-y| \leq t\},
\end{aligned}
$$

we have,

$$
\omega_{g}^{l, L}(t) \leq\|h\|_{L^{\infty}[l, L]} \cdot \omega_{v}^{l, L}(t)+\left\|g_{v}\right\|_{L^{\infty}[l, L]} \cdot \omega_{h}^{l, L}(t) .
$$

Again by long computations, we can to check that $g_{v}$ is a Dini continuous function with modulus of continuity satisfying a condition of the type (DTC). Precisely, there exists a constant $C(v)>0$, such that

$$
\int_{0}^{L-l} \frac{\omega_{v}^{l, L}(t)}{t} \mathrm{~d} t \leq C(v) \cdot \xi\left(\frac{L-l}{l}\right),
$$

where

$\xi(t):= \begin{cases}\left(\ln t^{-1}\right)^{1-2 v}+t, & \text { if } L-l \in\left(0, e^{-(2 v+1)}\right], l \in(0,1], \\ {\left[\ln \left(\frac{t^{-1}}{1+e^{-(2 v+1)}}\right)\right]^{1-2 v}+t,} & \text { if } L-l \in\left(0, e^{-(2 v+1)}\right], l \in\left(1,1+e^{-(2 v+1)}\right], \\ t, & \text { if } L-l \in\left(e^{-(2 v+1)}, \infty\right) \text { or } l \in\left(1+e^{-(2 v+1)}, \infty\right) .\end{cases}$

Finally, using the definitions of $g_{v}$ and $g$, the local Lipschitz regularity of $h$, estimate (9.6) and one more time by long and tedious computations we conclude that there exists $C^{*}(\nu)>0$, such that

$$
\omega_{g}^{l, L}(t) \leq C^{*}(v) \cdot\left(1+\frac{L}{l}\right)\left(\omega_{v}^{l, L}(t)+\frac{t}{l}\right)
$$


This example now follows by direct computation of the Dini condition by inequality above using (9.7).

We finish this section discussing about scaling. The estimates for minimizers of functionals considered here involve universal constants, i.e, depending, for instance, of $\delta$ and $g_{0}$. Many these estimates also depend on $G(1)$. In this case, it is very important that our minimizers admit rescaling that normalize the values $G(1)$. This fact is what allows us to run a compactness argument using the result above to perform a blow-up analysis in the proof of Theorem 2.2. For $s>0$ consider the following scalings

$$
G_{s}(t):=\frac{G(s t)}{s G^{\prime}(s)} \quad \text { and } \quad G_{s}^{*}(t):=G(s t) .
$$

We observe that this rescalings preserve regularity. Also, if $G \in \mathcal{G}\left(\delta, g_{0}\right) \Longrightarrow G_{s} \in$ $\mathcal{G}\left(\delta, g_{0},\left(1+g_{0}\right)^{-1}\right)$ and $G_{s}(1) \leq 1$. Still, $G \in \mathcal{G}\left(\delta, g_{0}, \varepsilon_{0}\right), s \geq 1 \Longrightarrow G_{s}^{*} \in \mathcal{G}\left(\delta, g_{0}, \varepsilon_{0}\right)$. In the next lemma, we show the invariance under this scaling of the Dini type control (DTC).

Lemma 9.2 (Scaling properties)

$$
G \in \mathcal{G}_{\left(\xi_{1}, \xi_{2}\right) \cdot D}\left(\delta, g_{0}\right) \Longrightarrow G_{s} \in \mathcal{G}_{\left(\xi_{1}, \xi_{2}\right) \cdot D}\left(\delta, g_{0},\left(1+g_{0}\right)^{-1}\right) \text { and } G_{s}(1) \leq 1 \text {. }
$$

$$
G \in \mathcal{G}_{\left(\xi_{1}, \xi_{2}\right) \cdot D}\left(\delta, g_{0}, \varepsilon_{0}\right), s \geq 1 \Longrightarrow G_{s}^{*} \in \mathcal{G}_{\left(\xi_{1}, \xi_{2}\right) \cdot D}\left(\delta, g_{0}, \varepsilon_{0}\right) .
$$

Proof Let $G \in \mathcal{G}\left(\delta, g_{0}\right)$. We see that $Q_{g_{s}}(t)=Q_{g_{s}^{*}}(t)=Q_{g}(s t), \forall t>0$. Using the Definition 2.7 we obtain $\omega_{g_{s}}^{l, L}(t)=\omega_{g_{s}^{*}}^{l, L}(t) \leq \omega_{g}^{s l, s L}(s t)$. Thus,

$$
\begin{aligned}
\int_{0}^{L-l} \frac{\omega_{g_{s}}^{l, L}(t)}{t} \mathrm{~d} t=\int_{0}^{L-l} \frac{\omega_{g_{s}^{*}}^{l, L}(t)}{t} \mathrm{~d} t & \leq \int_{0}^{L-l} \frac{\omega_{g}^{s l, s L}(s t)}{t} \mathrm{~d} t \\
& \leq \int_{0}^{s(L-l)} \frac{\omega_{g}^{s l, s L}(\zeta)}{\zeta} d \zeta \\
& \leq C_{1}^{*}\left(\delta, g_{0}\right) \cdot \xi_{1}\left(\frac{L}{l}\right) \cdot \xi_{2}\left(\frac{L-l}{l}\right)
\end{aligned}
$$

\section{The proof of Theorem 2.2}

In this moment, we discuss the proof of our main result. Before prove it, we enunciate a lemma presented in [6] that provides an estimate putting in perspective the Hölder and Lipschitz continuity character of functions. We observe that the method of the proof of Theorem 2.2 appears first in the papers $[8,14]$. We follow the same spirit of the Theorem 2.1 of [6]. For this reason, we present only the major steps.

Lemma 10.1 (Hölder/Lipschitz continuity character) Let $w:(0,1] \rightarrow \mathbb{R}$ be a nonnegative and nondecreasing function such that $w(1) \leq L$ for some $L>0$. Suppose $0<\tau<1$ and $0<\alpha \leq 1$ are such that

$$
w\left(\tau^{k+1}\right) \leq \max _{0 \leq m \leq k}\left\{L \cdot \tau^{\alpha(k+1)}, \tau^{\alpha(m+1)} \cdot w\left(\tau^{k-m}\right)\right\} \text { for every } k \geq 0 .
$$


Then,

$$
w(r) \leq \tau^{-\alpha} L r^{\alpha} \quad \text { for } 0<r \leq 1 .
$$

In particular, if $u$ is a bounded function in $B_{1}$ such that $\sup _{B_{1}}|u| \leq L$ and

$$
\sup _{B_{\tau^{k+1}}}|u| \leq \max _{0 \leq m \leq k}\left\{L \cdot \tau^{\alpha(k+1)}, \tau^{\alpha(m+1)} \cdot \sup _{B_{\tau^{k-m}}}|u|\right\} \text { for every } k \geq 0,
$$

we have the following estimate,

$$
|u(x)| \leq \tau^{-\alpha} L|x|^{\alpha} \quad \text { for every } \quad x \in B_{1} .
$$

Proof of Theorem 2.2 Without loss of generality, we can assume that $x_{0}=0$. By Lemma 10.1 , it is enough to show that there exists a constant $c_{0} \in(0,1)$ such that for any $u \in$ $S_{\varepsilon_{0}}^{\xi_{1}, \xi_{2}}\left(B_{1}, M\right)$ satisfying

$$
\min \left\{\Theta_{u}^{+}(0, r), \Theta_{u}^{-}(0, r)\right\} \leq c_{0}, \forall r \in(0,1)
$$

we have

$$
\sup _{B_{2^{-(k+1)}}}|u(x)| \leq \max _{0 \leq m \leq k}\left\{\frac{M}{c_{0} \cdot 2^{k+1}}, \frac{S(k-m)}{2^{m+1}}\right\}, \quad \forall k \geq 0
$$

where $S(j):=\sup _{B_{2^{-j}}}|u|$. So, let us suppose by contradiction, that (10.3) does not hold. Then, for each $j \in \mathbb{N}, j \geq 2$, we can find integers $k_{j} \geq 0$ and functions $u_{j} \in S_{\varepsilon_{0}}^{\xi_{1}, \xi_{2}}\left(B_{1}, M\right)$ such that

$$
\min \left\{\Theta_{u_{j}}^{+}\left(0,2^{-k_{j}}\right), \Theta_{u_{j}}^{-}\left(0,2^{-k_{j}}\right)\right\} \leq \frac{1}{j} \rightarrow 0 \text { as } j \rightarrow \infty
$$

but

$$
\sup _{B_{2}-\left(k_{j}+1\right)}\left|u_{j}(x)\right|>\max _{0 \leq m \leq k_{j}}\left\{\frac{j \cdot M}{2^{k_{j}+1}}, \frac{S_{j}\left(k_{j}-m\right)}{2^{m+1}}\right\} \text {, where } S_{j}\left(k_{j}-m\right)=\sup _{B_{2}-\left(k_{j}-m\right)}\left|u_{j}\right| .
$$

Because of (10.4) we can assume that, up to subsequence of $\left\{k_{j}\right\}$,

$$
\Theta_{u_{j}}^{-}\left(0,2^{-k_{j}}\right) \leq \frac{1}{j} \rightarrow 0 \text { as } j \rightarrow \infty .
$$

Moreover, (10.5) implies that $k_{j} \geq \log _{2} j-1 \rightarrow \infty$. Now we consider the following family of auxiliary functions

$$
v_{j}(x):=\frac{u_{j}\left(2^{-k_{j}} x\right)}{S_{j}\left(k_{j}+1\right)}, \quad x \in B_{2^{k_{j}}} .
$$

Since density is scaling invariant, (10.6) implies that $\left|\left\{v_{j}<0\right\} \cap B_{1}\right| \leq \frac{1}{j}\left|B_{1}\right|$. We also know that $u_{j}$ is a local minimizer of a functional

$$
\mathcal{J}_{G_{j}}(u, \Omega)=\int_{\Omega}\left[G_{j}(|\nabla u|)+f_{1, j}(x) H_{1, j}\left(u^{+}\right)+f_{2, j}(x) H_{2, j}\left(u^{-}\right)+Q\left(h_{1, j}, h_{2, j}\right)(u)(x)\right] \mathrm{d} x,
$$

satisfying the conditions of the Definition 2.3 and $G_{j} \in \mathcal{G}_{\left(\xi_{1}, \xi_{2}\right) \cdot D}\left(\delta, g_{0}, \varepsilon_{0}\right)$. It easy to check that $u_{j}$ minimizes a functional similar to (10.8) substituting the function $Q\left(h_{1, j}, h_{2, j}\right)(u)(x)$ by

$$
\widetilde{Q}\left(h_{1, j}, h_{2, j}\right)(u)(x)=\left(h_{2, j}-h_{1, j}\right) \chi_{\{u>0\}}-\left(h_{2, j}-h_{1, j}\right)^{-} \chi_{\{u=0\}} .
$$


Setting $g_{j}=G_{j}^{\prime}$, we can consider the following family of normalized rescalings

$$
\bar{G}_{j}(t):=G_{\sigma_{j}}(t)=\frac{G_{j}\left(\sigma_{j} t\right)}{\sigma_{j} g_{j}\left(\sigma_{j}\right)}, \quad \text { where } \sigma_{j}:=2^{k_{j}} \cdot S_{j}\left(k_{j}+1\right) .
$$

By Lemma 9.2 we have $\bar{G}_{j} \in \mathcal{G}_{\left(\xi_{1}, \xi_{2}\right) \cdot D}\left(\delta, g_{0},\left(1+g_{0}\right)^{-1}\right)$ and $\bar{G}_{j}(1) \leq 1$. Again by (10.5) follows that $\sigma_{j} \geq \frac{j}{2} \rightarrow \infty$. Now, we note that $v_{j}$ is a minimizer for the functional

$$
\begin{gathered}
\widetilde{\mathcal{J}}_{\bar{G}_{j}}(w, \Omega)=\int_{\Omega}\left[\bar{G}_{j}(|\nabla w|)+\bar{f}_{1, j}(x) \bar{H}_{1, j}\left(w^{+}\right)\right. \\
\left.+\bar{f}_{2, j}(x) \bar{H}_{2, j}\left(w^{-}\right)+\widetilde{Q}\left(\bar{h}_{1, j}, \bar{h}_{2, j}\right)(w)(x)\right] \mathrm{d} x,
\end{gathered}
$$

where

$\bar{f}_{i, j}(x)=\frac{f_{i, j}\left(2^{-k_{j}} x\right)}{\sigma_{j} g_{j}\left(\sigma_{j}\right)}, \quad \bar{H}_{i, j}\left(w^{ \pm}\right)=H_{i, j}\left(S_{j}\left(k_{j}+1\right) w^{ \pm}\right) \quad$ and $\quad \bar{h}_{i, j}(x)=\frac{h_{i, j}\left(2^{-k_{j}} x\right)}{\sigma_{j} g_{j}\left(\sigma_{j}\right)}$.

Once more by (10.5), $G_{j}(1) \geq \varepsilon_{0},(G-2)$ and since $\sigma_{j} \rightarrow \infty$, we conclude that

$$
\left\|v_{j}\right\|_{L^{\infty}\left(B_{8}\right)} \leq 16, \quad\left\|\bar{f}_{i, j}(x) \bar{H}_{i, j}\left(w^{ \pm}\right)\right\|_{L^{\infty}\left(B_{8}\right)} \rightarrow 0, \quad\left\|\widetilde{Q}\left(\bar{h}_{1, j}, \bar{h}_{2, j}\right)(w)\right\|_{L^{\infty}\left(B_{8}\right)} \rightarrow 0
$$

for any bounded function $w: B_{2^{k_{j}}} \rightarrow \mathbb{R}$. This imply that for $j$ large enough $v_{j} \in$ $\mathcal{S}_{\left(1+g_{0}\right)^{-1}}^{\xi_{1}, \xi_{2}}\left(B_{8}, 16\right)$. This way, the uniform Hölder estimate - Theorem 5.1 - implies that

$$
\left\|v_{j}\right\|_{C^{0, \alpha}\left(B_{4}\right)} \leq 16+C .
$$

So, we conclude that there exists a $v_{\infty} \in C^{0, \alpha}\left(B_{4}\right)$ such that $v_{j} \longrightarrow v_{\infty}$ uniformly in $B_{4}$. Now, considering $\left\{w_{j}\right\}_{j \geq 1}$ solutions in $W^{1, \bar{G}_{j}}\left(B_{4}\right)$ to the following Dirichlet problem

$$
\mathcal{L}_{j} w_{j}=0 \quad \text { in } B_{4} \text { and } w_{j}=v_{j} \quad \text { on } \partial B_{4},
$$

where the $\mathcal{L}_{j}$ 's are given by

$$
\mathcal{L}_{j} w:=\operatorname{div}\left(\bar{g}_{j}(|\nabla w|) \frac{\nabla w}{|\nabla w|}\right), \quad \bar{g}_{j}=\bar{G}_{j}^{\prime},
$$

we conclude similarly to the proof of Theorem 2.1 of [6] that

$$
h_{j}:=v_{j}-w_{j} \longrightarrow 0 \text { strongly in } W_{0}^{1, \delta+1}\left(B_{4}\right) .
$$

By Maximum Principle and (10.10) follow that $\left\|w_{j}\right\|_{L^{\infty}\left(B_{4}\right)}=\left\|v_{j}\right\|_{L^{\infty}\left(B_{4}\right)} \leq 16$.

Now, Theorem 3.1 guarantees that there exists $C \stackrel{\left(B_{4}\right)}{=} C\left(n, \delta, g_{0}\right)>0$ such that $\left\|w_{j}\right\|_{C^{1, \alpha}\left(B_{2}\right)} \leq C$. Therefore, we can find $w_{\infty} \in C^{1, \alpha / 2}\left(B_{2}\right)$ such that up to a subsequence

$$
w_{j} \longrightarrow w_{\infty}, \nabla w_{j} \longrightarrow \nabla w_{\infty} \text { uniformly in } B_{2} \text {. }
$$

We conclude this way that $v_{\infty}=w_{\infty}$ in $B_{2}$ by (10.11). Now, we can use our compactness result - Theorem 9.1 - to conclude that there exists a $G_{\infty} \in \mathcal{G}\left(\delta, g_{0}\right)$ such that, again up to a subsequence,

$$
\bar{G}_{j} \longrightarrow G_{\infty} \text { and } \bar{G}_{j}^{\prime} \longrightarrow G_{\infty}^{\prime} \text { uniformly in compact subsets of }[0, \infty),
$$

and

$$
\bar{G}_{j}^{\prime \prime} \longrightarrow G_{\infty}^{\prime \prime} \text { uniformly in compact subsets of }(0, \infty)
$$


By estimates (10.10) and convergence of $w_{j}$ and $\bar{G}_{j}$ follows that $v_{\infty}$ is a minimizer of $I\left(v, B_{1}\right):=\int_{B_{1}} G_{\infty}(|\nabla v|) \mathrm{d} x$. This implies that for $g_{\infty}:=G_{\infty}^{\prime}, v_{\infty}$ is a weak solution to

$$
\begin{aligned}
& \mathcal{L}_{\infty} v_{\infty}:=\operatorname{div}\left(g_{\infty}\left(\left|\nabla v_{\infty}\right|\right) \frac{\nabla v_{\infty}}{\left|\nabla v_{\infty}\right|}\right)=0 \quad \text { in } \quad B_{1}, \\
& \text { and } \quad v_{\infty}(0)=0 \text { and } 0 \leq v_{\infty} \leq 2 \text { and } \sup _{B_{1 / 2}} v_{\infty}=1 .
\end{aligned}
$$

Therefore, by Harnack inequality (Lemma 3.4), we have for $C_{0}=C_{0}\left(\delta, g_{0}, n\right)>0$

$$
1=\sup _{B_{1 / 2}} v_{\infty} \leq C_{0} \cdot v_{\infty}(0)=0,
$$

which is a contradiction. This way (10.3) holds. If instead of (10.6) we have $\Theta_{u_{j}}^{+}\left(0,2^{-k_{j}}\right) \leq \frac{1}{j}$ the proof follows by slightly modifications of the previous case to consider the auxiliary function $V_{j}=-v_{j}$. This finish the proof of Theorem.

We are ready to prove the scaled version of Theorem 2.2, i.e. Corollary 2.1. In particular, it shows the dependence between the Lipschitz constant of minimizers and the scale where the density starts to become small enough. We use this result for to prove Lipschitz regularity and also density estimates from below in the next section.

Proof of Corollary 2.1 We can assume that $x_{0}=0$. For $u \in S_{\varepsilon_{0}}^{\xi_{1}, \xi_{2}}\left(B_{r}, M\right)$ follows that $u$ is a minimizer of

$$
\mathcal{J}_{G}\left(u, B_{r}\right)=\int_{B_{r}}\left[G(|\nabla u|)+f_{1}(x) H_{1}\left(u^{+}\right)+f_{2}(x) H_{2}\left(u^{-}\right)+Q\left(h_{1}, h_{2}\right)(u)(x)\right] \mathrm{d} x,
$$

satisfying the Definition 2.3 and $G \in \mathcal{G}_{\left(\xi_{1}, \xi_{2}\right) \cdot D}\left(\delta, g_{0}, \varepsilon_{0}\right)$. Define $v(x)=u(r x), x \in B_{1}$. In this case, for

$$
0<r \leq r_{0}^{*}:=\min \left\{1,\left(\frac{\varepsilon_{0}}{\left(1+g_{0}\right) \cdot \max _{i=1,2}\left\{\lambda_{i}, \mu_{i}\right\}}\right)^{(1+\delta)^{-1}}\right\},
$$

we can show that $v \in S_{\left(1+g_{0}\right)^{-1}}^{\xi_{1}, \xi_{2}}\left(B_{1}, M\right)$ with $\lambda_{i}=\mu_{i}=1$ in this class. Indeed, by simple computations and using Lemma 9.2, we note that $v$ is a local minimizer of

$$
\begin{aligned}
& \mathcal{J}_{G_{r^{-1}}}\left(w, B_{1}\right)=\int_{B_{1}}\left[G_{r^{-1}}(|\nabla w|)+\frac{r}{g\left(r^{-1}\right)}\left(\tilde{f}_{1}(x) H_{1}\left(w^{+}\right)\right.\right. \\
& \left.\left.+\tilde{f}_{2}(x) H_{2}\left(w^{-}\right)+Q\left(\tilde{h}_{1}, \tilde{h}_{2}\right)(w)(x)\right)\right] \mathrm{d} x,
\end{aligned}
$$

where $\tilde{f}_{i}(x)=f_{i}(r x), \tilde{h}_{i}(x)=h_{i}(r x)$ for $x \in B_{1}$ and $i=1,2$,

and, by $(g-2)$ and $(G-2)$, we have for $r \leq r_{0}^{*}$ that

$$
r^{-1} g\left(r^{-1}\right) \geq G\left(r^{-1}\right) \geq \frac{\varepsilon_{0}}{1+g_{0}} r^{-(1+\delta)} \geq \max _{i=1,2}\left\{\lambda_{i}, \mu_{i}\right\} .
$$

Now, applying Theorem 2.2 to $v$ we obtain

$$
|v(x)| \leq \frac{2 M}{c_{0}^{*}} \cdot|x|, \quad \forall x \in B_{1}(0),
$$


provided

$$
\min \left\{\Theta_{v}^{+}\left(x_{0}, \rho\right), \Theta_{v}^{-}\left(x_{0}, \rho\right)\right\} \leq c_{0}^{*}
$$

for all $0<\rho<1$, where $c_{0}^{*}=c_{0}^{*}\left(n, \delta, g_{0}, \varepsilon_{0}, \wp_{i}, \rho_{i}, \gamma_{i}, M, \xi_{i}\right)>0$. The result follows translating this back in terms of $u$.

\section{Lipschitz regularity under a "small density criterion" and asymptotic behaviour of the free boundary}

In this final section, we investigate the optimal regularity for local minimizers for functionals of the type (2.2) and the local behaviour of the free boundary in the case where the minimizers are only locally Hölder continuous both as a consequence of Theorem 2.2. We start with the proof of Theorem 2.3.

Proof of Theorem 2.3 Consider $\Omega^{\prime} \Subset \Omega$ and $y_{0} \in \Omega^{\prime} \cap\{u \neq 0\}$. We can assume that $u\left(y_{0}\right)>0$ since the case $u\left(y_{0}\right)<0$ can be treated similarly. Define

$$
d_{y}^{+}:=\operatorname{dist}(y, \partial\{u>0\}) \quad \text { and } \quad d_{y}^{-}:=\operatorname{dist}(y, \partial\{u<0\}) .
$$

Assume first that $d_{y_{0}}^{-} \geq r_{\sigma}^{*} / 2$. In this case we have $0 \leq u \in \mathcal{S}_{\varepsilon_{0}}\left(B_{r_{\sigma}^{*} / 2}\left(y_{0}\right), M, \Lambda_{i}^{M}\right)$. By Theorem 6.1,

$$
\left|\nabla u\left(y_{0}\right)\right| \leq \frac{4 \cdot C_{2}}{r_{\sigma}^{*}} .
$$

Suppose now $d_{y_{0}}^{-}<r_{\sigma}^{*} / 2$. Let $z_{0} \in F^{+}(u)$ such that $d_{y_{0}}^{+}=\left|y_{0}-z_{0}\right|$. Since also $d_{y_{0}}^{+}<r_{\sigma}^{*} / 2$ we conclude that $d_{z_{0}}^{-}<r_{\sigma}^{*}$. Thus, Corollary 2.1 assure us

$$
u\left(y_{0}\right) \leq \frac{2 M}{c_{0} \cdot r_{\sigma}^{*}} \cdot d_{y_{0}}^{+} .
$$

By Corollary 5.1, $\mathcal{L}_{g} u=H_{1}^{\prime}(u) f_{1}$ in $B_{d_{y_{0}}^{+}}\left(y_{0}\right)$, thus by Harnack inequality and above estimate, we obtain

$$
u(x) \leq C_{3}\left(\frac{2 M}{c_{0} \cdot r_{\sigma}^{*}}+g^{-1}\left(d_{y_{0}}^{+} \Lambda_{1}^{M} \cdot \lambda_{1}\right)\right) \cdot d_{y_{0}}^{+}, \quad \forall x \in B_{\frac{d_{y_{0}}^{+}}{2}}\left(y_{0}\right) .
$$

Now, by local gradient estimate (3.3),

$$
\left|\nabla u\left(y_{0}\right)\right| \leq C_{4}\left[C_{3}\left(\frac{2 M}{c_{0} \cdot r_{\sigma}^{*}}+g^{-1}\left(d_{y_{0}}^{+} \Lambda_{1}^{M} \cdot \lambda_{1}\right)\right)+g^{-1}\left(d_{y_{0}}^{+} \Lambda_{1}^{M} \cdot \lambda_{1}\right)\right] \leq \frac{C_{5}}{r_{\sigma}^{*}} .
$$

In the sequel, we investigate the touching behaviour of the free boundaries $F^{+}(u)$ and $F^{-}(u)$ even though local minima $u$ are only Hölder continuous. Notice that in the classical paper [4], suitable conditions imply that minimizers are globally subharmonic functions (see Theorem 2.3 in [4]) and this fact together with the maximum principle restrict the way the free boundaries $F^{+}(u), F^{-}(u)$ may touch. Essentially $F^{-}(u)$ cannot separate from $F^{+}(u)$. In other words, the set $F^{-}(u) \backslash F^{+}(u)$ is empty. In this generality treated here, minimizers 
are neither subsolutions nor supersolutions and the negative free boundary may, in principle, separate from the positive one, i.e,

$$
\left(F^{+}(u) \backslash F^{-}(u)\right) \cap \Omega \neq \varnothing .
$$

A similar situation occurs even in the standard case involving the Laplace operator in a inhomogeneous setting as it appears in flame propagation problems in [13].

As a second application of Theorem 2.2, we can show that if Lipschitz regularity fails to be the optimal regularity for a minimizer $u \in S_{\varepsilon_{0}}^{\xi_{1}, \xi_{2}}(\Omega, M)$ say in a point, then this point is a contact point between the free boundaries and the positive and negative phase of $u$ have a universal (upper) density from below at that point. In particular, in this contact point the free boundary $F^{ \pm}(u)$ is cusp free. In the case where $u$ is not locally Lipschitz around $x_{0}$, then $x_{0}$ is also a contact point between the free boundaries and $F^{ \pm}(u)$ is "asymptotically cusp free". The proof follows the same guide lines of Proposition 2.1 of [6].

Proof of Theorem 2.4 Let $\Omega^{\prime} \Subset \Omega$. It is easy to see that $\mathcal{S}_{p}\left(\Omega^{\prime}\right) \subset \mathcal{S}_{l}\left(\Omega^{\prime}\right)$. Now consider $x_{0} \in \mathcal{S}_{l}\left(\Omega^{\prime}\right)$. We can find two sequences $\left\{x_{n}\right\}_{n \geq 1},\left\{y_{n}\right\}_{n \geq 1} \subset \Omega^{\prime}$ such that:

$$
x_{n} \rightarrow x_{0}, y_{n} \rightarrow x_{0} \quad \text { as } n \rightarrow \infty \quad \text { and } \quad \rho_{n}:=\frac{\left|u\left(x_{n}\right)-u\left(y_{n}\right)\right|}{\left|x_{n}-y_{n}\right|}>n .
$$

Note that $\Omega=\{u>0\} \cup\{u<0\} \cup\{u=0\}^{\circ} \cup F^{ \pm}(u)$ and by interior $C^{1, \alpha}$-regularity we conclude that

$$
x_{0} \notin\{u>0\} \cup\{u<0\} \cup\{u=0\}^{\circ} .
$$

Thus, $x_{0} \in F^{ \pm}(u)$. Let us suppose that $x_{0} \in F^{+}(u) \backslash F^{-}(u)$. In this case, $x_{0} \notin F^{-}(u) \cap \overline{\Omega^{\prime}}$ which is a compact set. Hence, for $0<\varepsilon=\min \left\{\frac{3}{4} d_{x_{0}}^{-}\right.$, dist $\left.\left(\Omega^{\prime}, \partial \Omega\right)\right\}$, we have that $B_{\varepsilon}\left(x_{0}\right) \cap F^{-}(u) \cap \overline{\Omega^{\prime}}=\varnothing$. Since $0 \leq u \in S_{\varepsilon_{0}}^{\left(\xi_{1}, \xi_{2}\right) \cdot D}\left(B_{\varepsilon}\left(x_{0}\right), M\right)$, we have by one-phase case (Theorem 6.1) that

$$
n<\frac{\left|u\left(x_{n}\right)-u\left(x_{0}\right)\right|}{\left|x_{n}-x_{0}\right|} \leq\|\nabla u\|_{L^{\infty}\left(B_{\varepsilon / 2}\left(x_{0}\right)\right)} \leq \frac{C}{\varepsilon}<\infty,
$$

which is a contradiction. Similarly we treat the case where $x_{0} \in F^{-}(u) \backslash F^{+}(u)$. Hence, $x_{0} \in F^{+}(u) \cap F^{-}(u)$. Now we verify (2.8) and (2.9). Consider first $x_{0} \in \mathcal{S}_{l}\left(\Omega^{\prime}\right)$. By Theorem 2.3, we know that for any $r \in\left(0, r_{1}^{*}\right)$ there exists $x_{r} \in B_{\frac{3 r}{4}}\left(x_{0}\right) \cap F^{ \pm}(u)$ such that for some $\rho_{r} \in(0, r / 4)$ we have

$$
\min \left\{\Theta_{u}^{+}\left(x_{r}, \rho_{r}\right), \Theta_{u}^{-}\left(x_{r}, \rho_{r}\right)\right\}>c_{0}^{*} .
$$

This proves (2.8). Suppose now that $x_{0} \in \mathcal{S}_{p}\left(\Omega^{\prime}\right)$, we also see that for any $0<r \leq r_{1}^{*}$ there exists $0<\rho \leq r$ such that

$$
\min \left\{\Theta_{u}^{+}\left(x_{0}, \rho\right), \Theta_{u}^{-}\left(x_{0}, \rho\right)\right\}>c_{0}^{*} .
$$

This follows by Corollary 2.1. Thus, (2.9) is proved.

Remark 11.1 By above Theorem we obtain that

$$
F^{ \pm}(u)=\mathcal{S}_{p}\left(\Omega^{\prime}\right) \dot{\bigcup}\left(\mathcal{S}_{l}\left(\Omega^{\prime}\right) \backslash \mathcal{S}_{p}\left(\Omega^{\prime}\right)\right) \dot{\bigcup}\left(F^{ \pm}(u) \backslash \mathcal{S}_{l}\left(\Omega^{\prime}\right)\right) .
$$


In the set $\mathcal{S}_{p}\left(\Omega^{\prime}\right)$, the free boundary is cusp free and, in the set $\mathcal{S}_{l}\left(\Omega^{\prime}\right) \backslash \mathcal{S}_{p}\left(\Omega^{\prime}\right)$, asymptotically cusp free. Finally, we note that for any $z_{0} \in F^{ \pm}(u) \backslash \mathcal{S}_{l}\left(\Omega^{\prime}\right)$ there exists $r_{0} \in\left(0, r_{1}^{*}\right)$ such that

$$
[u]_{C^{0,1}\left(B_{r}\left(z_{0}\right)\right)} \leq C^{*}, \quad r \in\left(0, r_{0}\right] .
$$

In this case, if $z_{0} \in F^{+}(u)$, by Lipschitz continuity and strong non-degeneracy (see Theorem 4.1 of [16] or Theorem 5.1 of [18]) there exists a constant $c_{1}^{*} \in(0,1)$ depending only on intrinsic parameters and $C^{*}$ such that

$$
\left(1-c_{1}^{*}\right) \cdot r^{n} \geq\left|B_{r}\left(z_{0}\right) \cap\{u>0\}\right| \geq c_{1}^{*} \cdot r^{n}, \quad r \in\left(0, r_{0}\right) .
$$

Furthermore, following a similar strategy of the Lemma 4.2 and Theorem 4.3 of [16], there exist positive constants $c_{2}^{*} \leq C_{2}^{*}<\infty$ such that

$$
c_{2}^{*} \cdot r^{n-1} \leq\left|B_{r}\left(z_{0}\right) \cap F^{+}(u)\right| \leq C_{2}^{*} \cdot r^{n-1} .
$$

The two above estimates follows similarly in the case where $z_{0} \in F^{-}(u)$. This proves that the open set $F^{ \pm}(u) \backslash \mathcal{S}_{l}\left(\Omega^{\prime}\right)$ (with respect to the topology induced by $F^{ \pm}(u)$ ) is cusp free and has locally finite perimeter in each connected component.

Acknowledgements The author would like to thank the anonymous referee for the suitable comments and suggestions and also for pointing out some corrections in the original version.

\section{References}

1. Adams, R., Fournier, J.: Sobolev Spaces, 2nd edn. Academic Press, Cambridge (2003)

2. Alt, H.W., Caffarelli, L.A.: Existence and regularity for a minimum problem with free boundary. J. Reine Angew. Math. 325, 105-144 (1981)

3. Alt, H.W., Caffarelli, L.A., Friedman, A.: Free boundary problem for quasi-linear elliptic equations. Institiut für Angewandte Mathematik 1-44 (1983)

4. Alt, H.W., Caffarelli, L.A., Friedman, A.: Variational problems with two phases and their free boundaries. Trans. Am. Math. Soc. 282, 431-461 (1984)

5. Azzam, J., Bedrossian, J.: Bounded mean oscillation and the uniqueness of active scalar equations. Trans. Am. Math. Soc. 367, 3095-3118 (2015)

6. Braga, J., Ederson, M., Moreira, D.: Uniform Lipschitz regularity for classes of minimizers in two phase free boundary problems in Orlicz spaces with small density on the negative phase. Ann. Inst. H. Poincaré Anal. Non Linéaire 31(4), 823-850 (2014)

7. Caffarelli, L.A.: A Harnack inequality approach to the regularity of free boundaries. III. Existence theory, compactness, and dependence on X. Ann. Sc. Norm. Super. Pisa Cl. Sci. 15(4), 583-602 (1989)

8. Caffarelli, L.A., Karp, L., Shahgholian, H.: Regularity of a free boundary with application to the Pompeiu problem. Ann. Math. 151, 269-292 (2000)

9. Danielli, D., Petrosyan, A.: A minimum problem with free boundary for a degenerate quasilinear operator. Calc. Var. Partial Differ. Equ. 23(1), 97-124 (2005)

10. Giusti, E.: Direct Methods in the Calculus of Variations. World Scientific Publishing Co., Inc., River Edge, NJ (2003)

11. Kharakhanyan, A.: On the Lipschitz regularity of solutions of a minimum problem with free boundary. Interfaces Free Bound. 10, 79-86 (2008)

12. Ladyzhenskaya, O.A., Ural'tseva, N.N.: Linear and Quasilinear Elliptic Equations. Mathematics in Science and Engineering, vol. 46. Academic Press, New York (1968)

13. Lederman, C., Wolanski, N.: A two phase elliptic singular perturbation problem with a forcing term. J. Math. Pures Appl. (9) 86(6), 552-589 (2006)

14. Lee, K., Shahgholian, H.: Regularity of a free boundary for viscosity solutions of nonlinear elliptic equations. Commun. Pure Appl. Math. LIV, 43-56 (2001)

15. Leitão, R., Queiroz, O.S., Teixeira, E.: Regularity for two-phase free boundary problems. Ann. Inst. H. Poincaré Anal. Non Linéaire 32(4), 741-762 (2015) 
16. Leitão, R.J., Teixeira, E.V.: Regularity and geometric estimates for minima of discontinuous functionals. Rev. Mat. Iberoam. 31(1), 69-108 (2015)

17. Lieberman, G.M.: The natural generalization of the natural conditions of Ladyzhensaya and Uraltseva for elliptic equations. Commun. Partial Differ. Equ. 16(2 \& 3), 311-361 (1991)

18. Martinez, S., Wolanski, N.: A minimum problem with free boundary in Orlicz spaces. Adv. Math. 218(6), 1914-1971 (2008)

19. Moreira, D., Wang, L.: Hausdorff measure estimates and Lipschitz regularity in inhomogeneous nonlinear free boundary problems. Arch. Ration. Mech. Anal. 213, 527-559 (2014)

20. Zheng, J., Zhang, Z., Zhao, P.: A minimum problem with two-phase free boundary in Orlicz spaces. Monatsh. Math. 172, 441-475 (2013) 